\title{
Best approximations of the $\phi$-Hadamard fractional Volterra integro-differential equation by matrix valued fuzzy control functions
}

\author{
Safoura Rezaei Aderyani ${ }^{1 *}$ and Reza Saadati ${ }^{1}$
}

"Correspondence:

safoura_rezaei99@mathdep.iust.ac.ir 'Department of Mathematics, Iran

University of Science and

Technology, Tehran, Iran

\section{Springer}

\begin{abstract}
In this article, first, we present an example of fuzzy normed space by means of the Mittag-Leffler function. Next, we extend the concept of fuzzy normed space to matrix valued fuzzy normed space and also we introduce a class of matrix valued fuzzy control functions to stabilize a nonlinear $\phi$-Hadamard fractional Volterra integro-differential equation. In this sense, we investigate the Ulam-Hyers-Rassias stability for this kind of fractional equations in matrix valued fuzzy Banach space. Finally, as an application, we investigate the Ulam-Hyers-Rassias stability using matrix valued fuzzy control function obtained through the Mittag-Leffler function.
\end{abstract}

MSC: Primary 28A80; 47H10; secondary $46 \mathrm{~S} 10$

Keywords: $\phi$-Hadamard fractional equation; Volterra integro-differential equation; MVFB-space; Ulam-Hyers-Rassias stability; Fixed point method

\section{Introduction and preliminaries}

Fractional Calculus (FC) is considered as a branch of mathematical analysis which deals with the investigation and applications of integrals and derivatives of arbitrary order. Therefore, FC is an extension of the integer-order calculus that considers integrals and derivatives of any real or complex order [1], i.e., unifying and generalizing the notions of integer-order differentiation and $n$-fold integration.

Ulam-Hyers stability is one of the main topics in the theory of functional equations. Generally a functional equation is said to be stable provided that, for any function $f$ satisfying the perturbed functional equation, there exists an exact solution $f_{0}$ of that equation which is not far from the given $f$. Based on this concept, the study of the stability of functional equations can be regarded as a branch of optimization theory [2].

The origin of Ulam stability theory was an open problem formulated by Ulam, in 1940, concerning the stability of homomorphism. The first partial answer to Ulam's question came within a year, when Hyers proved a stability result, for the additive Cauchy equation in Banach spaces. The result of the stability of Cauchy equations was further gen-

(c) The Author(s) 2021. This article is licensed under a Creative Commons Attribution 4.0 International License, which permits use, sharing, adaptation, distribution and reproduction in any medium or format, as long as you give appropriate credit to the original author(s) and the source, provide a link to the Creative Commons licence, and indicate if changes were made. The images or other third party material in this article are included in the article's Creative Commons licence, unless indicated otherwise in a credit line to the material. If material is not included in the article's Creative Commons licence and your intended use is not permitted by statutory regulation or exceeds the permitted use, you will need to obtain permission directly from the copyright holder. To view a copy of this licence, visit http://creativecommons.org/licenses/by/4.0/. 
eralized by Rassias. The first result on Ulam-Hyers stability of differential equations was given by Obloza. Alsina and Ger investigated the stability of differential equations $y^{\prime}=y$. The results of Alsina and Ger were extended by many authors to the stability of the first order linear differential equation and linear differential equations of higher order [3].

Here, we let $\mathcal{G}_{1}=[0, \xi]$, with $\xi>0, \mathcal{G}_{2}=(0, \infty), \mathcal{G}_{3}=(0,1], \mathcal{G}_{4}=[0, \infty]$ and $\mathcal{G}_{5}=[0,1]$ (note $\mathcal{G}_{5}^{\circ}=(0,1)$ denotes the interior of $\mathcal{G}_{5}$ ).

Suppose $\mathcal{W}$ is a vector space and $\zeta_{0} \in \mathcal{G}_{2}$. We denote the set of fuzzy set (in short, F-set) by $\mathcal{Y}$. Now $\mathscr{Y} \in \mathcal{Y}$ means $\mathscr{Y}: \mathcal{W} \times \mathcal{G}_{2} \rightarrow \mathcal{G}_{3}$ satisfies the following conditions:

$\left(\mathcal{C}_{1}\right) \mathscr{Y}$ is continuous;

$\left(\mathcal{C}_{2}\right) \mathscr{Y}(\omega, \cdot)$ is non-decreasing, where $\omega \in \mathcal{W}$;

$\left(\mathcal{C}_{3}\right) \lim _{\zeta_{0} \rightarrow+\infty} \mathscr{Y}\left(\omega, \zeta_{0}\right)=1$, where $\omega \in \mathcal{W}$.

Definition 1.1 ([4-6]) A continuous binary operation $*: \mathcal{G}_{5} \times \mathcal{G}_{5} \rightarrow \mathcal{G}_{5}$ with the following condition is said to be a continuous triangular norm (in short, CTN) if;

( ا) $\mathcal{F} * \mathcal{H}=\mathcal{H} * \mathcal{F}$ and $\mathcal{F} *(\mathcal{P} * \mathcal{H})=(\mathcal{F} * \mathcal{P}) * \mathcal{H}$ for all $\mathcal{F}, \mathcal{H}, \mathcal{P} \in \mathcal{G}_{5}$;

(ıl) $\mathcal{F} * 1=\mathcal{F}$ for all $\mathcal{F} \in \mathcal{G}_{5}$;

(ılı) $\mathcal{F} * \mathcal{P} \leq \mathcal{F}^{\prime} * \mathcal{P}^{\prime}$ when $\mathcal{F} \leq \mathcal{F}^{\prime}$ and $\mathcal{P} \leq \mathcal{P}^{\prime}$ for every $\mathcal{F}, \mathcal{F}^{\prime}, \mathcal{P}, \mathcal{P}^{\prime} \in \mathcal{G}_{5}$.

Here we present some CTNs.

$\left(\mathcal{E}_{1}\right) \mathcal{F} * \mathscr{P} \mathcal{H}=\mathcal{F H}(:$ the product $\mathrm{CTN})$;

$\left(\mathcal{E}_{2}\right) \mathcal{F} * \mathscr{M} \mathcal{H}=\wedge\{\mathcal{F}, \mathcal{H}\}$ (: the minimum CTN);

( $\left.\mathcal{E}_{3}\right) \mathcal{F} * \mathscr{L} \mathcal{H}=\vee\{\mathcal{F}+\mathcal{H}-1,0\}$ (: the Lukasiewicz CTN). Note that due to the continuity of $*$, the above axioms (characterizing general triangular norms) can be relaxed, i.e., it is enough to require the associativity, 0 to be an annihilator of $*, \mathcal{F} * 0=0 * \mathcal{F}=0$ for any $\mathcal{F} \in \mathcal{G}_{5}$, and 1 is an idempotent element of $*, 1 * 1=1$. For more details see [5].

Definition 1.2 ([7]) Consider CTN $*$, the vector space $\mathcal{W}$ and the F-set $\mathscr{L}: \mathcal{W} \times \mathcal{G}_{2} \rightarrow \mathcal{G}_{3}$. Now $(\mathcal{W}, \mathscr{L}, *)$ is called a fuzzy normed space if:

$(\mathcal{L} 1) \mathscr{L}\left(\omega, \zeta_{\circ}\right)>0$ for every $\zeta_{\circ} \in \mathcal{G}_{2}$;

$(\mathcal{L} 2) \quad \mathscr{L}\left(\omega, \zeta_{\circ}\right)=1$ for every $\zeta_{\circ} \in \mathcal{G}_{2}$ if and only if $\omega=0$;

$(\mathcal{L} 3) \mathscr{L}\left(\hbar \omega, \zeta_{\circ}\right)=\mathscr{L}\left(\omega, \frac{\zeta_{\circ}}{|\hbar|}\right)$ for every $\omega \in \mathcal{W}$ and $\hbar \in \mathbb{C}$ with $\hbar \neq 0$;

$(\mathcal{L} 4) \quad \mathscr{L}\left(\omega+\omega^{\prime}, \zeta_{\circ}+\zeta_{0}^{\prime}\right) \geq \mathscr{L}\left(\omega, \zeta_{0}\right) * \mathscr{L}\left(\omega^{\prime}, \zeta_{\circ}^{\prime}\right)$ for every $\omega, \omega^{\prime} \in \mathcal{W}$ and $\zeta_{\circ}, \zeta_{0}^{\prime} \in \mathcal{G}_{2}$.

Now we will present an example of fuzzy normed space by means of Mittag-Leffler function, but before that we introduce the concept of Mittag-Leffler function.

The special function

$$
\mathbf{E}_{c}(z)=\sum_{k=0}^{\infty} \frac{z^{k}}{\Im(1+c k)}, \quad c \in \mathbb{C}, \Re(c)>0, z \in \mathbb{C},
$$

and its general form

$$
\mathbf{E}_{c, d}(z)=\sum_{k=0}^{\infty} \frac{z^{k}}{\Im(d+c k)}, \quad c, d \in \mathbb{C}, \Re(c)>0, \Re(d)>0, z \in \mathbb{C},
$$


are called Mittag-Leffler function, in which $\mathbb{C}$ and $\Im$ are, respectively, the set of complex numbers and the Gamma function.

Consider the one-parameter Mittag-Leffler function

$$
\mathbf{E}_{c}\left(-\frac{\|\omega\|}{\zeta_{\circ}}\right)=\sum_{k=0}^{\infty} \frac{\left(-\frac{\|\omega\|}{\zeta_{0}}\right)^{k}}{\Im(1+c k)}, \quad c \in \mathcal{G}_{3}, \omega \in \mathcal{W}, \zeta_{0} \in \mathcal{G}_{2}
$$

Here, we want to show in the following four steps that $\left(\mathcal{W}, \mathbf{E}_{c}\left(-\frac{\|\omega\|}{\zeta_{0}}\right), * \mathscr{M}\right)$ is a fuzzy normed space.

$\left(\mathcal{L}_{1}\right)$ If $c \in \mathcal{G}_{3}$, then $\mathbf{E}_{c}(0)=1$ and $\lim _{\omega \rightarrow-\infty} \mathbf{E}_{c}(\omega)=0$, therefore we can conclude that $\mathbf{E}_{c}$ is an increasing function for all $c \in \mathcal{G}_{3}$, and also we have $\mathbf{E}_{c} \in \mathcal{G}_{3}$.

$\left(\mathcal{L}_{2}\right)$ It is straightforward to show $\mathbf{E}_{c}\left(-\frac{\|\omega\|}{\zeta_{0}}\right)=1$ for every $\zeta_{0} \in \mathcal{G}_{2}$, if and only if $\omega=0$.

$\left(\mathcal{L}_{3}\right)$ For any $\omega \in \mathcal{W}$ and $\zeta_{0} \in \mathcal{G}_{2}$, we have

$$
\begin{aligned}
\mathbf{E}_{c}\left(-\frac{\|\hbar \omega\|}{\zeta_{0}}\right) & =\sum_{k=0}^{\infty} \frac{\left(-\frac{\|\hbar \omega\|}{\zeta_{0}}\right)^{k}}{\Im(1+c k)} \\
& =\sum_{k=0}^{\infty} \frac{\left(-\frac{\|\omega\|}{\frac{\zeta_{0}}{\hbar \hbar}}\right)^{k}}{\Im(1+c k)} \\
& =\mathbf{E}_{c}\left(-\frac{\|\omega\|}{\frac{\zeta_{0}}{|\hbar|}}\right) .
\end{aligned}
$$

$\left(\mathcal{L}_{4}\right)$ Let $\mathbf{E}_{c}\left(-\frac{\|\omega\|}{\zeta_{0}}\right) \leq \mathbf{E}_{c}\left(-\frac{\left\|\omega^{\prime}\right\|}{\zeta_{0}^{\prime}}\right)$. Then we have $\frac{\left\|\omega^{\prime}\right\|}{\zeta_{0}^{\prime}} \leq \frac{\|\omega\|}{\zeta_{0}}$, for any $\omega, \omega^{\prime} \in \mathcal{W}$ and $\zeta_{0}, \zeta_{0}^{\prime} \in$ $\mathcal{G}_{2}$. Now, if $\omega=\omega^{\prime}$, we have $\zeta_{0} \leq \zeta_{0}^{\prime}$. Thus, otherwise, we have

$$
\begin{aligned}
\frac{\|\omega\|}{\zeta_{0}}+\frac{\|\omega\|}{\zeta_{0}} & \geq \frac{\|\omega\|}{\zeta_{0}}+\frac{\left\|\omega^{\prime}\right\|}{\zeta_{0}^{\prime}} \\
& \geq 2 \frac{\|\omega\|}{\zeta_{0}+\zeta_{0}^{\prime}}+2 \frac{\left\|\omega^{\prime}\right\|}{\zeta_{0}+\zeta_{0}^{\prime}} \\
& \geq 2 \frac{\left\|\omega+\omega^{\prime}\right\|}{\zeta_{0}+\zeta_{0}^{\prime}},
\end{aligned}
$$

therefore $\frac{\|\omega\|}{\zeta_{0}} \geq \frac{\left\|\omega+\omega^{\prime}\right\|}{\zeta_{0}+\zeta_{0}^{\prime}}$. But $-\frac{\|\omega\|}{\zeta_{0}} \leq-\frac{\left\|\omega+\omega^{\prime}\right\|}{\zeta_{0}+\zeta_{0}^{\prime}}$, and also

$$
\sum_{k=0}^{\infty} \frac{\left(-\frac{\|\omega\|}{\zeta_{\circ}}\right)^{k}}{\Im(1+c k)} \leq \sum_{k=0}^{\infty} \frac{\left(-\frac{\left\|\omega+\omega^{\prime}\right\|}{\zeta_{\circ}+\zeta_{0}^{\prime}}\right)^{k}}{\Im(1+c k)}
$$

which implies that

$$
\mathbf{E}_{c}\left(-\frac{\|\omega\|}{\zeta_{0}}\right) \leq \mathbf{E}_{c}\left(-\frac{\left\|\omega+\omega^{\prime}\right\|}{\zeta_{0}+\zeta_{0}^{\prime}}\right)
$$

Hence we have

$$
\mathbf{E}_{c}\left(-\frac{\left\|\omega+\omega^{\prime}\right\|}{\zeta_{0}+\zeta_{0}^{\prime}}\right) \geq \min \left\{\mathbf{E}_{c}\left(-\frac{\|\omega\|}{\zeta_{0}}\right), \mathbf{E}_{c}\left(-\frac{\left\|\omega^{\prime}\right\|}{\zeta_{0}^{\prime}}\right)\right\},
$$


for any $\omega, \omega^{\prime} \in \mathcal{W}$ and $\zeta_{0}, \zeta_{0}^{\prime} \in \mathcal{G}_{2}$. Therefore

$$
\mathscr{L}\left(\omega, \zeta_{0}\right)=\mathbf{E}_{c}\left(-\frac{\|\omega\|}{\zeta_{0}}\right)
$$

defines a fuzzy norm and $(\mathcal{W}, \mathscr{L}, * \mathscr{M})$ is a fuzzy normed space, for any $\omega \in \mathcal{W}, \zeta_{\circ} \in \mathcal{G}_{2}$ and $c \in \mathcal{G}_{3}$; here $(\mathcal{W},\|\cdot\|)$ is a normed linear space.

Now we extend the concept of triangular norms mentioned above and $[4,6]$ on $\operatorname{diag} \mathcal{M}_{n}\left(\mathcal{G}_{5}\right)$.

Let

$$
\operatorname{diag} \mathcal{M}_{n}\left(\mathcal{G}_{5}\right)=\left\{\left[\begin{array}{lll}
g_{1} & & \\
& \ddots & \\
& & g_{n}
\end{array}\right]=\operatorname{diag}\left[g_{1}, \ldots, g_{n}\right], g_{1}, \ldots, g_{n} \in \mathcal{G}_{5}\right\},
$$

where $\operatorname{diag} \mathcal{M}_{n}\left(\mathcal{G}_{5}\right)$ is equipped with the partial order relation:

$$
\begin{aligned}
& \boldsymbol{g}:=\operatorname{diag}\left[g_{1}, \ldots, g_{n}\right], \quad \boldsymbol{h}:=\operatorname{diag}\left[h_{1}, \ldots, h_{n}\right] \in \operatorname{diag} \mathcal{M}_{n}\left(\mathcal{G}_{5}\right), \\
& \boldsymbol{g} \preceq \boldsymbol{h} \Longleftrightarrow g_{j} \leq h_{j} \quad \text { for every } j=1, \ldots, n .
\end{aligned}
$$

Also, $\boldsymbol{g} \prec \boldsymbol{h}$ denotes that $\boldsymbol{g} \preceq \boldsymbol{h}$ and $\boldsymbol{g} \neq \boldsymbol{h} ; \boldsymbol{g} \ll \boldsymbol{h}$ and $g_{j}<h_{j}$ for every $j=1, \ldots, n$. We define $\varrho:=\operatorname{diag}[\varrho, \ldots, \varrho]$ in $\operatorname{diag} \mathcal{M}_{n}\left(\mathcal{G}_{5}\right)$ where $\varrho \in \mathcal{G}_{5}$. For example, $\mathbf{1}=\operatorname{diag}[1, \ldots, 1]$ and $\mathbf{0}=\operatorname{diag}[0, \ldots, 0]$.

Definition 1.3 A generalized triangular norm (in short, GTN) on $\operatorname{diag} \mathcal{M}_{n}\left(\mathcal{G}_{5}\right)$ is an operation $\circledast: \operatorname{diag} \mathcal{M}_{n}\left(\mathcal{G}_{5}\right) \times \operatorname{diag} \mathcal{M}_{n}\left(\mathcal{G}_{5}\right) \rightarrow \operatorname{diag} \mathcal{M}_{n}\left(\mathcal{G}_{5}\right)$ satisfying the following conditions:

( $) \quad\left(\forall \boldsymbol{g} \in \operatorname{diag} \mathcal{M}_{n}\left(\mathcal{G}_{5}\right)\right)(\boldsymbol{g} \circledast \mathbf{1}=\boldsymbol{g})$ (boundary condition);

( ıl) $\left(\forall(\boldsymbol{g}, \boldsymbol{h}) \in\left(\operatorname{diag} \mathcal{M}_{n}\left(\mathcal{G}_{5}\right)\right)^{2}\right)(\boldsymbol{g} \circledast \boldsymbol{h}=\boldsymbol{h} \circledast \boldsymbol{g})$ (commutativity);

( $ı$ li) $\left(\forall(\boldsymbol{g}, \boldsymbol{h}, \boldsymbol{v}) \in\left(\operatorname{diag} \mathcal{M}_{n}\left(\mathcal{G}_{5}\right)\right)^{3}\right)(\boldsymbol{g} \circledast(\boldsymbol{h} \circledast \boldsymbol{v})=(\boldsymbol{g} \circledast \boldsymbol{h}) \circledast \boldsymbol{v})$ (associativity);

$($ iv $)\left(\forall(\boldsymbol{g}, \boldsymbol{h}, \boldsymbol{v}, \boldsymbol{k}) \in\left(\operatorname{diag} \mathcal{M}_{n}\left(\mathcal{G}_{5}\right)\right)^{4}\right)(\boldsymbol{g} \preceq \boldsymbol{h}$; and $\boldsymbol{v} \preceq \boldsymbol{k} \Longrightarrow \boldsymbol{g} \circledast \boldsymbol{v} \preceq \boldsymbol{h} \circledast \boldsymbol{k})$ (monotonicity).

For every $\boldsymbol{g}, \boldsymbol{h} \in \operatorname{diag} \mathcal{M}_{n}\left(\mathcal{G}_{5}\right)$ and every sequences $\left\{\boldsymbol{g}_{k}\right\}$ and $\left\{\boldsymbol{h}_{k}\right\}$ converging to $\boldsymbol{g}$ and $\boldsymbol{h}$ suppose we have

$$
\lim _{k}\left(\boldsymbol{g}_{k} \circledast \boldsymbol{h}_{k}\right)=\boldsymbol{g} \circledast \boldsymbol{h},
$$

then $\circledast$ on $\operatorname{diag} \mathcal{M}_{n}\left(\mathcal{G}_{5}\right)$ is continuous GTN (in short, CGTN). Now we present some examples of CGTN.

$(\mathcal{E} 1) \quad$ Define $\circledast \mathscr{M}: \operatorname{diag} \mathcal{M}_{n}\left(\mathcal{G}_{5}\right) \times \operatorname{diag} \mathcal{M}_{n}\left(\mathcal{G}_{5}\right) \rightarrow \operatorname{diag} \mathcal{M}_{n}\left(\mathcal{G}_{5}\right)$, such that

$$
\begin{aligned}
\boldsymbol{g} \circledast \mathscr{M} \boldsymbol{h} & =\operatorname{diag}\left[g_{1}, \ldots, g_{n}\right] \circledast \mathscr{M} \operatorname{diag}\left[h_{1}, \ldots, h_{n}\right] \\
& =\operatorname{diag}\left[\min \left\{g_{1}, h_{1}\right\}, \ldots, \min \left\{g_{n}, h_{n}\right\}\right],
\end{aligned}
$$

then $\circledast \mathscr{M}$ is CGTN (minimum CGTN). 
$(\mathcal{E} 2)$ Define $\circledast \mathscr{P}: \operatorname{diag} \mathcal{M}_{n}\left(\mathcal{G}_{5}\right) \times \operatorname{diag} \mathcal{M}_{n}\left(\mathcal{G}_{5}\right) \rightarrow \operatorname{diag} \mathcal{M}_{n}\left(\mathcal{G}_{5}\right)$, such that

$$
\boldsymbol{g} \circledast \mathscr{P} \boldsymbol{h}=\operatorname{diag}\left[g_{1}, \ldots, g_{n}\right] \circledast \mathscr{P} \operatorname{diag}\left[h_{1}, \ldots, h_{n}\right]=\operatorname{diag}\left[g_{1} \cdot h_{1}, \ldots, g_{n} \cdot h_{n}\right],
$$

then $\circledast \mathscr{P}$ is CGTN (product CGTN).

$(\mathcal{E} 3)$ Define $\circledast \mathscr{L}: \operatorname{diag} \mathcal{M}_{n}\left(\mathcal{G}_{5}\right) \times \operatorname{diag} \mathcal{M}_{n}\left(\mathcal{G}_{5}\right) \rightarrow \operatorname{diag} \mathcal{M}_{n}\left(\mathcal{G}_{5}\right)$, such that

$$
\begin{aligned}
\boldsymbol{g} \circledast \mathscr{L} \boldsymbol{h} & =\operatorname{diag}\left[g_{1}, \ldots, g_{n}\right] \circledast \mathscr{L} \operatorname{diag}\left[h_{1}, \ldots, h_{n}\right] \\
& =\operatorname{diag}\left[\max \left\{g_{1}+h_{1}-1,0\right\}, \ldots, \max \left\{g_{n}+h_{n}-1,0\right\}\right],
\end{aligned}
$$

then $\circledast \mathscr{L}$ is CGTN (Lukasiewicz CGTN).

Note that $\prod_{j=1}^{n} g_{j}=g_{1} \circledast \cdots \circledast g_{n}$, for $g_{1}, \ldots, g_{n} \in \mathcal{G}_{5}$ and $\circledast \mathscr{M}=\bigwedge$. Now we present some numerical examples:

$$
\begin{aligned}
& \operatorname{diag}\left[\frac{6}{7}, \frac{7}{9}, \frac{3}{4}, 1\right] \circledast \mathscr{M} \operatorname{diag}\left[\frac{2}{5}, 0, \frac{4}{7}, \frac{3}{8}\right]
\end{aligned}
$$

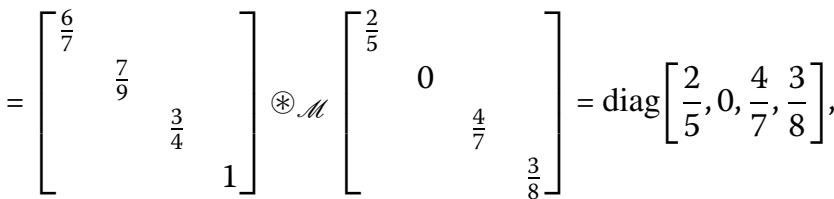

$$
\begin{aligned}
& \operatorname{diag}\left[\frac{6}{7}, \frac{7}{9}, \frac{3}{4}, 1\right] \circledast \mathscr{P} \operatorname{diag}\left[\frac{2}{5}, 0, \frac{4}{7}, \frac{3}{8}\right] \\
& =\left[\begin{array}{llll}
\frac{6}{7} & & & \\
& \frac{7}{9} & & \\
& & \frac{3}{4} & \\
& & & 1
\end{array}\right] \circledast \mathscr{P}\left[\begin{array}{cccc}
\frac{2}{5} & & & \\
& 0 & & \\
& & \frac{4}{7} & \\
& & & \frac{3}{8}
\end{array}\right]=\operatorname{diag}\left[\frac{12}{35}, 0, \frac{12}{28}, \frac{3}{8}\right] \text {, } \\
& \operatorname{diag}\left[\frac{6}{7}, \frac{7}{9}, \frac{3}{4}, 1\right] \circledast \mathscr{L} \operatorname{diag}\left[\frac{2}{5}, 0, \frac{4}{7}, \frac{3}{8}\right]
\end{aligned}
$$

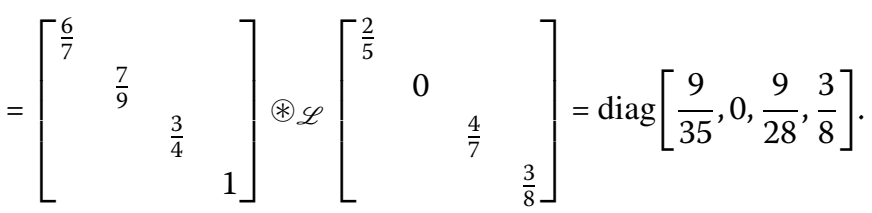

Then we get

$$
\begin{aligned}
& \operatorname{diag}\left[\frac{6}{7}, \frac{7}{9}, \frac{3}{4}, 1\right] \circledast \mathscr{M} \operatorname{diag}\left[\frac{2}{5}, 0, \frac{4}{7}, \frac{3}{8}\right] \\
& \succeq \operatorname{diag}\left[\frac{6}{7}, \frac{7}{9}, \frac{3}{4}, 1\right] \circledast \mathscr{P} \operatorname{diag}\left[\frac{2}{5}, 0, \frac{4}{7}, \frac{3}{8}\right] \\
& \succeq \operatorname{diag}\left[\frac{6}{7}, \frac{7}{9}, \frac{3}{4}, 1\right] \circledast \mathscr{L} \operatorname{diag}\left[\frac{2}{5}, 0, \frac{4}{7}, \frac{3}{8}\right] .
\end{aligned}
$$

Suppose $\mathcal{W}$ is a vector space and $\vec{\zeta} \in\left(\mathcal{G}_{2}\right)^{n}$ for $n \in \mathbb{N}, \vec{\zeta}=\left(\zeta_{1}, \ldots, \zeta_{n}\right)$, in which $\zeta_{j} \in \mathcal{G}_{2}$ for all $j=1, \ldots, n$. Note that $\overrightarrow{0} \prec \vec{\zeta}$ if and only if $0<\zeta_{j}, \forall j=1, \ldots, n$ and $\vec{\zeta} \longrightarrow \infty$ is equivalent to $\zeta_{j} \rightarrow \infty$, for all $j=1, \ldots, n$. 
We denote the set of matrix valued fuzzy set (MVF-set) by $\Psi$. Now $\psi \in \Psi$ means that $\psi: \mathcal{W} \times\left(\mathcal{G}_{2}\right)^{n} \rightarrow \operatorname{diag} \mathcal{M}_{n}\left(\mathcal{G}_{3}\right)$ satisfies the following conditions:

$(\mathcal{C} 1) \psi$ is continuous;

$(\mathcal{C} 2) \psi(\omega, \cdot)$ is non-decreasing, where $\omega \in \mathcal{W}$;

(C3) $\lim _{\vec{\zeta} \rightarrow+\infty} \psi(\omega, \vec{\zeta})=\mathbf{1}$, where $\omega \in \mathcal{W}$.

In $\Psi$ we define $\preceq$ as follows:

$$
\psi \precsim \chi \quad \Longleftrightarrow \quad \psi(\omega, \vec{\zeta}) \preceq \chi\left(\omega, \overrightarrow{\zeta^{\prime}}\right), \quad \forall \omega \in \mathcal{W} \text { and } \vec{\zeta}, \overrightarrow{\zeta^{\prime}} \in\left(\mathcal{G}_{2}\right)^{n}
$$

Definition 1.4 Consider the CGTN $\circledast$, a vector space $\mathcal{W}$ and MVF-set $\mathscr{S}: \mathcal{W} \times\left(\mathcal{G}_{2}\right)^{n} \rightarrow$ $\operatorname{diag} \mathcal{M}_{n}\left(\mathcal{G}_{3}\right)$. In this case, we define a matrix valued fuzzy normed space (MVFN-space) $(\mathcal{W}, \mathscr{S}, \circledast)$ as

(S1) $\mathscr{S}(\omega, \vec{\zeta})=\mathbf{1}$, for all $\vec{\zeta} \succ \overrightarrow{0}$ if and only if $w=0$;

$(\mathcal{S} 2) \mathscr{S}(\hbar \omega, \vec{\zeta})=\mathscr{S}\left(\omega, \frac{\vec{\zeta}}{|\hbar|}\right)$ for all $\omega \in \mathcal{W}, \vec{\zeta} \succ \overrightarrow{0}$ and $\hbar \in \mathbb{C}$ with $\hbar \neq 0$;

(S3) $\mathscr{S}\left(\omega+\omega^{\prime}, \vec{\zeta}+\overrightarrow{\zeta^{\prime}}\right) \succeq \mathscr{S}(\omega, \vec{\zeta}) \circledast \mathscr{S}\left(w^{\prime}, \overrightarrow{\zeta^{\prime}}\right)$ for all $\omega, \omega^{\prime} \in \mathcal{W}$ and $\vec{\zeta}, \overrightarrow{\zeta^{\prime}} \succ \overrightarrow{0}$.

(S4) $\lim _{\vec{\zeta} \rightarrow+\infty} \mathscr{S}(\omega, \vec{\zeta})=\mathbf{1}$, for all $\omega \in \mathcal{W}$.

A complete MVFN-space is called matrix valued fuzzy Banach space (or MVFB-space). For example the MVF-set $\mathscr{S}$,

$$
\mathscr{S}(\omega, \vec{\zeta})=\operatorname{diag}\left[\mathbf{E}_{\aleph}\left(-\frac{\|\omega\|}{\zeta_{1}}\right), \frac{\zeta_{2}}{\zeta_{2}+\|\omega\|}, \exp \left(-\frac{\|\omega\|}{\zeta_{3}}\right), \mathbf{E}_{\aleph}\left(-\frac{\|\omega\|}{\zeta_{4}}\right)\right]
$$

is a matrix valued fuzzy norm, where $\vec{\zeta} \in\left(\mathcal{G}_{2}\right)^{4}, \mathbf{E}_{\aleph}, \aleph \in \mathcal{G}_{3}$, is the one-parameter Mittag Leffler function and $(\mathcal{W}, \mathscr{S}, \circledast \mathscr{M})$ is an MVFN-space; here $(\mathcal{W},\|\cdot\|)$ is a normed linear space.

The approximation of functional equations was studied in MVFN-spaces, fuzzy metric spaces and random multi-normed space [8]. Also stability results for stochastic fractional differential and integral equations were considered in [2, 3, 9-14].

Theorem 1.5 (Alternative theorem $[15,16])$ Let $(\Phi, \delta)$ be a complete $\mathcal{G}_{4}$-valued metric space and let $\Gamma: \Phi \rightarrow \Phi$ be a strictly contractive function with the Lipschitz constant $\wp<1$. Then, for a given element $\varphi \in \Phi$, either

$$
\delta\left(\Gamma^{\tau} \varphi, \Gamma^{\tau+1} \varphi\right)=\infty
$$

for each $\tau \in \mathbb{N}$ or there is $\tau_{0} \in \mathbb{N}$ such that

(i) $\delta\left(\Gamma^{\tau} \varphi, \Gamma^{\tau+1} \varphi\right)<\infty$, for every $\tau \geq \tau_{0}$;

(ii) the fixed point $\mho^{*}$ of $\Gamma$ is the convergent point of the sequence $\left\{\Gamma^{\tau} \varphi\right\}$;

(iii) in the set $\Phi^{*}=\left\{\mho \in \Phi \mid \delta\left(\Gamma^{\tau_{0}} \varphi, \mho\right)<\infty\right\}, \mho^{*}$ is the unique fixed point of $\Gamma$;

(iv) $(1-\wp) \delta\left(\mho, \mho^{*}\right) \leq \delta(\mho, \Gamma \mho)$ for every $\mho \in \Phi^{*}$.

\section{$2 \phi$-Hadamard fractional equations}

In this section, we begin by introducing the definitions of the $\phi$-Hadamard-type fractional integral and the $\phi$-Hadamard-type fractional derivative of Caputo type.

Finally, we introduce the concept of stability of Ulam-Hyers-Rassias (in short, UHR). 
Definition 2.1 Let $(\alpha, \beta)$ be an interval on the real line $\mathbb{R}$, and $\phi(\varsigma)$ be a non-decreasing and positive monotone function on $(\alpha, \beta]$, having a continuous derivative $\phi^{\prime}(\varsigma)$ (denote first derivative as $\frac{d}{d \varsigma} \phi(\varsigma)$ on $\left.(\alpha, \beta)\right)$. The left-side $\phi$-Hadamard-type fractional integral with order $\kappa>0$ and parameter $\vartheta>0$, of an integrable function $\eta(\varsigma)$, regarding $\phi(\varsigma)$, on $[\alpha, \beta]$ is defined as

$$
{ }_{\alpha^{+}}^{H} \mathcal{I}_{\phi(\varsigma)}^{\kappa, \vartheta} \eta(\varsigma)=\frac{1}{\Im(\kappa)} \int_{\alpha}^{\varsigma} \mathcal{Q}(\varsigma, \iota) \eta(\iota) d \iota,
$$

where $\mathcal{Q}(\varsigma, \iota)=\left(\frac{\phi(\iota)}{\phi(\varsigma)}\right)^{\vartheta}\left(\log \frac{\phi(\varsigma)}{\phi(\iota)}\right)^{\kappa-1} \frac{\phi^{\prime}(l)}{\phi(l)}, \varsigma \in[\alpha, \beta]$ and $\Im$ is the Gamma function.

Definition 2.2 ([17]) Let $n-1<\kappa<n$ with $n \in \mathbb{N}$. Let $\mathcal{J}=[\alpha, \beta]$ be an interval such that $-\infty \leq \alpha<\beta \leq \infty$, and let $\eta, \phi \in C^{n}[\alpha, \beta]$ be two functions such that $\phi$ is non-decreasing and $\phi^{\prime}(\varsigma) \neq 0$, for all $\varsigma \in \mathcal{J}$. The $\phi$-Hadamard-type fractional derivative of Caputo type ${ }_{\alpha^{+}}^{H C} \mathcal{D}_{\phi(\varsigma)}^{\kappa, \vartheta} \eta(\varsigma)$ of a function $\eta$ of order $\kappa>0$ and parameter $\vartheta>0$ is defined as

$$
{ }_{\alpha^{+}}^{H C} \mathcal{D}_{\phi(\varsigma)}^{\kappa, \vartheta} \eta(\varsigma)={ }_{\alpha^{+}}^{H} \mathcal{I}_{\phi(\varsigma)}^{(n-\kappa), \vartheta}{ }_{\alpha^{+}}^{H} \mathcal{D}_{\phi(\varsigma)}^{n, \vartheta} \eta(\varsigma), \quad \varsigma \in[\alpha, \beta]
$$

where

$$
{ }_{\alpha^{+}}^{H} \mathcal{D}_{\phi(\varsigma)}^{n, \vartheta} \eta(\varsigma)=\phi(\varsigma)^{-\vartheta}\left(\frac{\phi(\varsigma)}{\phi^{\prime}(\varsigma)} \cdot \frac{d}{d \varsigma}\right)^{n}\left[\phi(\varsigma)^{\vartheta} \eta(\varsigma)\right] .
$$

Consider the $\phi$-Hadamard fractional Volterra integro-differential equation, defined by

$$
{ }_{0^{+}}^{H C} \mathcal{D}_{\phi(\varsigma)}^{\kappa, \vartheta} \eta(\varsigma)=\mu(\varsigma, \eta(\varsigma))+\int_{0}^{\varsigma} \mathcal{K}(\varsigma, \iota, \eta(\iota)) d \iota,
$$

where $\kappa \in \mathcal{G}_{5}^{\circ}, \vartheta \in \mathcal{G}_{5}$ and $\mu: \mathcal{G}_{1} \times \mathcal{W} \rightarrow \mathcal{W}, \mathcal{K}: \mathcal{G}_{1} \times \mathcal{G}_{1} \times \mathcal{W} \rightarrow \mathcal{W}$.

Let $\psi: \mathcal{W} \times\left(\mathcal{G}_{2}\right)^{n} \rightarrow \operatorname{diag} \mathcal{M}_{n}\left(\mathcal{G}_{3}\right)$ be a matrix valued fuzzy control function. The equation (2.3) is said to be UHR stable if $\eta(\varsigma)$ is a given differentiable function, satisfying

$$
\mathscr{S}\left({ }_{0^{+}}^{H C} \mathcal{D}_{\phi(\varsigma)}^{\kappa, \vartheta} \eta(\varsigma)-\mu(\varsigma, \eta(\varsigma))-\int_{0}^{\varsigma} \mathcal{K}(\varsigma, \iota, \eta(\iota)) d \iota, \vec{\zeta}\right) \succeq \psi(\varsigma, \vec{\zeta})
$$

for $\varsigma \in \mathcal{G}_{5}$, and we can find a solution $\eta^{\prime}(\varsigma)$ of equation (2.3) such that, for some $\partial>0$,

$$
\mathscr{S}\left(\eta(\varsigma)-\eta^{\prime}(\varsigma), \vec{\zeta}\right) \succeq \psi\left(\varsigma, \frac{\vec{\zeta}}{\partial}\right)
$$

Using the fixed point technique (Alternative theorem), we study HUR stability of the $\phi$-Hadamard fractional Volterra integro-differential equation (2.3) in MVFB-space $(\mathcal{W}, \mathscr{S}, \circledast)$. Our results can apply to improve recent results [17] and by methods used in this paper we can extend some fractional Volterra integro-differential equations in MVFBspaces [18-20]. 


\section{Best approximation of a $\phi$-Hadamard fractional Volterra integro-differential} equation

In this section, we apply a fixed point technique derived from Theorem 1.5 to study HUR stability of functional equation (2.3) for more details we refer to [21, 22]. Consider the MVFB-space $(\mathcal{W}, \mathscr{S}, \circledast)$ and MVF-set $\psi: \mathcal{W} \times\left(\mathcal{G}_{2}\right)^{n} \rightarrow \operatorname{diag} \mathcal{M}_{n}\left(\mathcal{G}_{3}\right)$. We set

$$
\Phi:=\left\{\eta: \mathcal{G}_{1} \rightarrow \mathcal{W}, \eta \text { is differentiable }\right\}
$$

and define a mapping $\delta$ from $\Phi \times \Phi$ to $\mathcal{G}_{4}$ by

$$
\begin{aligned}
\delta\left(\eta, \eta^{\prime}\right) & =\inf \left\{\lambda \in \mathcal{G}_{2}: \mathscr{S}\left(\begin{array}{c}
H C \\
0^{+}
\end{array} \mathcal{D}_{\phi(\varsigma)}^{\kappa, \vartheta} \eta(\varsigma)-{ }_{0^{+}}^{H C} \mathcal{D}_{\phi(\varsigma)}^{\kappa, \vartheta} \eta^{\prime}(\varsigma), \vec{\zeta}\right) \circledast \mathscr{M} \mathscr{S}\left(\eta(\varsigma)-\eta^{\prime}(\varsigma), \vec{\zeta}\right)\right. \\
& \left.\succeq \psi\left(\varsigma, \frac{\vec{\zeta}}{\lambda}\right), \forall \eta, \eta^{\prime} \in \Phi, \varsigma \in \mathcal{G}_{1}, \vec{\zeta} \in\left(\mathcal{G}_{2}\right)^{n}\right\} .
\end{aligned}
$$

Theorem $3.1(\Phi, \delta)$ is a complete $\mathcal{G}_{4}$-valued metric space.

Proof First, we show that $(\Phi, \delta)$ is a $\mathcal{G}_{4}$-valued metric space.

We show that $\delta\left(\eta, \eta^{\prime}\right)=0$ if and only if $\eta=\eta^{\prime}$. Let $\delta\left(\eta, \eta^{\prime}\right)=0$. Then we have

$$
\begin{aligned}
& \inf \left\{\lambda \in \mathcal{G}_{2}: \mathscr{S}\left({ }_{0^{+}}^{H C} \mathcal{D}_{\phi(\varsigma)}^{\kappa, \vartheta} \eta(\varsigma)-{ }_{0^{+}}^{H C} \mathcal{D}_{\phi(\varsigma)}^{\kappa, \vartheta} \eta^{\prime}(\varsigma), \vec{\zeta}\right) \circledast \mathscr{M} \mathscr{S}\left(\eta(\varsigma)-\eta^{\prime}(\varsigma), \vec{\zeta}\right)\right. \\
& \left.\quad \succeq \psi\left(\varsigma, \frac{\vec{\zeta}}{\lambda}\right), \forall \eta, \eta^{\prime} \in \Phi, \varsigma \in \mathcal{G}_{1}, \vec{\zeta} \in\left(\mathcal{G}_{2}\right)^{n}\right\}=0
\end{aligned}
$$

and so

$$
\mathscr{S}\left({ }_{0^{+}}^{H C} \mathcal{D}_{\phi(\varsigma)}^{\kappa, \vartheta} \eta(\varsigma)-{ }_{0^{+}}^{H C} \mathcal{D}_{\phi(\varsigma)}^{\kappa, \vartheta} \eta^{\prime}(\varsigma), \vec{\zeta}\right) \circledast \mathscr{M} \mathscr{S}\left(\eta(\varsigma)-\eta^{\prime}(\varsigma), \vec{\zeta}\right) \succeq \psi\left(\varsigma, \frac{\vec{\zeta}}{\lambda}\right)
$$

for all $\lambda \in \mathcal{G}_{2}$. Letting $\lambda$ tend to zero in the above inequality, we get

$$
\mathscr{S}\left({ }_{0^{+}}^{H C} \mathcal{D}_{\phi(\varsigma)}^{\kappa, \vartheta} \eta(\varsigma)-{ }_{0^{+}}^{H C} \mathcal{D}_{\phi(\varsigma)}^{\kappa, \vartheta} \eta^{\prime}(\varsigma), \vec{\zeta}\right) \circledast \mathscr{M} \mathscr{S}\left(\eta(\varsigma)-\eta^{\prime}(\varsigma), \vec{\zeta}\right)=1
$$

and so

$$
\mathscr{S}\left(\eta(\varsigma)-\eta^{\prime}(\varsigma), \vec{\zeta}\right)=1
$$

thus $\eta(\varsigma)=\eta^{\prime}(\varsigma)$ for every $\varsigma \in \mathcal{G}_{1}$, and vice versa. It is straightforward to show $\delta\left(\eta, \eta^{\prime}\right)=$ $\delta\left(\eta^{\prime}, \eta\right)$ for every $\eta, \eta^{\prime} \in \Phi$. Now let $\delta(\eta, \rho)=\ell_{1} \in \mathcal{G}_{2}$ and $\delta\left(\rho, \eta^{\prime}\right)=\ell_{2} \in \mathcal{G}_{2}$. Then we have

$$
\mathscr{S}\left({ }_{0^{+}}^{H C} \mathcal{D}_{\phi(\varsigma)}^{\kappa, \vartheta} \eta(\varsigma)-{ }_{0^{+}}^{H C} \mathcal{D}_{\phi(\varsigma)}^{\kappa, \vartheta} \rho(\varsigma), \vec{\zeta}\right) \circledast \mathscr{M} \mathscr{S}(\eta(\varsigma)-\rho(\varsigma), \vec{\zeta}) \succeq \psi\left(\varsigma, \frac{\vec{\zeta}}{\ell_{1}}\right)
$$

and

$$
\mathscr{S}\left({ }_{0^{+}}^{H C} \mathcal{D}_{\phi(\varsigma)}^{\kappa, \vartheta} \rho(\varsigma)-{ }_{0^{+}}^{H C} \mathcal{D}_{\phi(\varsigma)}^{\kappa, \vartheta} \eta^{\prime}(\varsigma), \vec{\zeta}\right) \circledast \mathscr{M} \mathscr{S}\left(\rho(\varsigma)-\eta^{\prime}(\varsigma), \vec{\zeta}\right) \succeq \psi\left(\varsigma, \frac{\vec{\zeta}}{\ell_{2}}\right) .
$$


Then we have

$$
\begin{aligned}
& \mathscr{S}\left({ }_{0^{+}}^{H C} \mathcal{D}_{\phi(\varsigma)}^{\kappa, \vartheta} \eta(\varsigma)-{ }_{0^{+}}^{H C} \mathcal{D}_{\phi(\varsigma)}^{\kappa, \vartheta} \eta^{\prime}(\varsigma),\left(\ell_{1}+\ell_{2}\right) \vec{\zeta}\right) \circledast \mathscr{M} \mathscr{S}\left(\eta(\varsigma)-\eta^{\prime}(\varsigma),\left(\ell_{1}+\ell_{2}\right) \vec{\zeta}\right) \\
& \geq {\left[\mathscr{S}\left(\begin{array}{l}
H C \\
0^{+}
\end{array} \mathcal{D}_{\phi(\varsigma)}^{\kappa, \vartheta} \eta(\varsigma)-{ }_{0^{+}}^{H C} \mathcal{D}_{\phi(\varsigma)}^{\kappa, \vartheta} \rho(\varsigma), \ell_{1} \vec{\zeta}\right)\right.} \\
&\left.\circledast \mathscr{M} \mathscr{S}\left({ }_{0^{+}}^{H C} \mathcal{D}_{\phi(\varsigma)}^{\kappa, \vartheta} \rho(\varsigma)-{ }_{0^{+}}^{H C} \mathcal{D}_{\phi(\varsigma)}^{\kappa, \vartheta} \eta^{\prime}(\varsigma), \ell_{2} \vec{\zeta}\right)\right] \\
& \circledast \mathscr{M}\left[\mathscr{S}\left(\eta(\varsigma)-\rho(\varsigma), \ell_{1} \vec{\zeta}\right) \circledast \mathscr{M} \mathscr{S}\left(\rho(\varsigma)-\eta^{\prime}(\varsigma), \ell_{2} \vec{\zeta}\right)\right] \\
& \geq \psi(\varsigma, \vec{\zeta}) \circledast \mathscr{M} \psi(\varsigma, \vec{\zeta}) \\
&= \psi(\varsigma, \vec{\zeta}),
\end{aligned}
$$

and so $\delta\left(\eta, \eta^{\prime}\right) \leq \ell_{1}+\ell_{2}$. Thus, $\delta\left(\eta, \eta^{\prime}\right) \leq \delta(\eta, \rho)+\delta\left(\rho, \eta^{\prime}\right)$. Now we are ready to prove $(\Phi, \delta)$ is complete. Let $\left\{\eta_{k}\right\}_{k}$ be a Cauchy sequence in $(\Phi, \delta)$. Let $\varsigma \in \mathcal{G}_{1}$ be fixed. Assume that $\sigma \in\left(\mathcal{G}_{2}\right)^{n}$ and $\Omega \in \mathcal{G}_{5}^{\circ}$ are arbitrary and consider $\vec{\zeta} \in\left(\mathcal{G}_{2}\right)^{n}$ such that $\psi(\varsigma, \vec{\zeta}) \succ 1-\Omega$. For $\varepsilon \vec{\zeta}<\vec{\sigma}$ choose $k^{\prime \prime} \in \mathbb{N}$ such that

$$
\delta\left(\eta_{k}, \eta_{k^{\prime}}\right)<\varepsilon, \quad \forall k, k^{\prime} \geq k^{\prime \prime}
$$

Then

$$
\begin{aligned}
\mathscr{S} & \left(\begin{array}{l}
H C \\
0^{+}
\end{array} \mathcal{D}_{\phi(\varsigma)}^{\kappa, \vartheta} \eta_{k}(\varsigma)-{ }_{0^{+}}^{H C} \mathcal{D}_{\phi(\varsigma)}^{\kappa, \vartheta} \eta_{k^{\prime}}(\varsigma), \vec{\sigma}\right) \circledast \mathscr{M} \mathscr{S}\left(\eta(\varsigma)-\eta^{\prime}(\varsigma), \vec{\sigma}\right) \\
& \succeq \mathscr{S}\left({ }_{0^{+}}^{H C} \mathcal{D}_{\phi(\varsigma)}^{\kappa, \vartheta} \eta_{k}(\varsigma)-{ }_{0^{+}}^{H C} \mathcal{D}_{\phi(\varsigma)}^{\kappa, \vartheta} \eta_{k^{\prime}}(\varsigma), \varepsilon \vec{\zeta}\right) \circledast \mathscr{M} \mathscr{S}\left(\eta(\varsigma)-\eta^{\prime}(\varsigma), \varepsilon \vec{\zeta}\right) \\
& \succeq \psi(\varsigma, \vec{\zeta}) \\
& \succ 1-\Omega .
\end{aligned}
$$

Then

$$
\mathscr{S}\left({ }_{0^{+}}^{H C} \mathcal{D}_{\phi(\varsigma)}^{\kappa, \vartheta} \eta_{k}(\varsigma)-{ }_{0^{+}}^{H C} \mathcal{D}_{\phi(\varsigma)}^{\kappa, \vartheta} \eta_{k^{\prime}}(\varsigma), \vec{\sigma}\right) \succ 1-\Omega
$$

and

$$
\mathscr{S}\left(\eta_{k}(\varsigma)-\eta_{k^{\prime}}(\varsigma), \vec{\sigma}\right) \succ 1-\Omega
$$

i.e., the sequence both $\left\{\eta_{k}(\varsigma)\right\}_{k}$ and $\left\{\left\{_{0^{+}}^{H C} \mathcal{D}_{\phi(\varsigma)}^{\kappa, \vartheta} \eta_{k}(\varsigma)\right\}_{k}\right.$ are Cauchy in complete space $(\mathcal{W}, \mathscr{S}, \circledast)$ on compact set $\mathcal{G}_{1}$, so they are uniformly convergent to the mapping $\eta: \mathcal{G}_{1} \rightarrow$ $\mathcal{W}$ and ${ }_{0^{+}}^{H C} \mathcal{D}_{\phi(\varsigma)}^{\kappa, \vartheta} \eta$, respectively. The uniform convergence leads us to the fact that $\eta$ is differentiable, i.e., an element of $\Phi$ and then $(\Phi, \delta)$ is complete.

Now we are ready to study UHR stability of the $\phi$-Hadamard fractional Volterra integrodifferential equation (2.3) and get the best approximation with better estimate for the pseudo- $\phi$-Hadamard fractional Volterra integro-differential equation. 
Theorem 3.2 Let $(\mathcal{W}, \mathscr{S}, \circledast)$ be an MVFB-space and $\Theta_{1}, \Theta_{2}, \Theta_{3}, \Theta_{4}$ and $\xi$ be positive constant such that $\max \left[\Theta_{1}, \Theta_{2} \Theta_{3}, \Theta_{1} \Theta_{4}, \Theta_{2} \Theta_{3} \Theta_{4}\right]<0.5$. Assume that the continuous mappings $\mu: \mathcal{G}_{1} \times \mathcal{W} \rightarrow \mathcal{W}, \mathcal{K}: \mathcal{G}_{1} \times \mathcal{G}_{1} \times \mathcal{W} \rightarrow \mathcal{W}$ with MVF-set $\psi: \mathcal{W} \times\left(\mathcal{G}_{2}\right)^{n} \rightarrow$ $\operatorname{diag} \mathcal{M}_{n}\left(\mathcal{G}_{3}\right)$ satisfy

$$
\begin{aligned}
& \mathscr{S}\left(\mu(\varsigma, \eta(\varsigma))-\mu\left(\varsigma, \eta^{\prime}(\varsigma)\right), \vec{\zeta}\right) \succeq \mathscr{S}\left(\eta(\varsigma)-\eta^{\prime}(\varsigma), \frac{\vec{\zeta}}{\Theta_{1}}\right), \\
& \mathscr{S}\left(\mathcal{K}(\varsigma, \iota, \eta(\iota))-\mathcal{K}\left(\varsigma, \iota, \eta^{\prime}(\iota)\right), \vec{\zeta}\right) \succeq \mathscr{S}\left(\eta(\iota)-\eta^{\prime}(\iota), \frac{\vec{\zeta}}{\Theta_{2}}\right), \quad \iota \leq \varsigma, \\
& \inf _{\delta \in \mathcal{G}_{1}} \psi(\delta, \vec{\zeta}) \succeq \psi\left(\varsigma, \frac{\xi \vec{\zeta}}{\Theta_{3}}\right),
\end{aligned}
$$

and

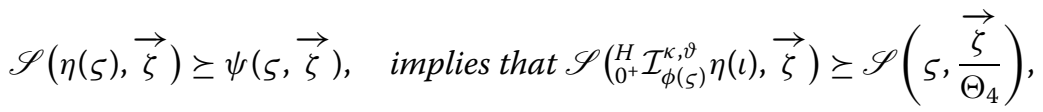

for every $\varsigma \in \mathcal{G}_{1}, \eta, \eta^{\prime}: \mathcal{G}_{1} \rightarrow \mathcal{W}$ and $\vec{\zeta} \in\left(\mathcal{G}_{2}\right)^{n}$. Let $\gamma: \mathcal{G}_{1} \rightarrow \mathcal{W}$ be a differentiable function satisfying

$$
\mathscr{S}\left({ }_{0^{+}}^{H C} \mathcal{D}_{\phi(\varsigma)}^{\kappa, \vartheta} \gamma(\varsigma)-\mu(\varsigma, \gamma(\varsigma))-\int_{0}^{\varsigma} \mathcal{K}(\varsigma, \iota, \gamma(\iota)) d \iota, \vec{\zeta}\right) \succeq \psi(\varsigma, \vec{\zeta}),
$$

for every $\varsigma \in \mathcal{G}_{1}$ and $\vec{\zeta} \in\left(\mathcal{G}_{2}\right)^{n}$. Then we have a unique differentiable function $\gamma_{0}: \mathcal{G}_{1} \rightarrow \mathcal{W}$ such that

$$
{ }_{0^{+}}^{H C} \mathcal{D}_{\phi(\varsigma)}^{\kappa, \vartheta} \gamma_{0}(\varsigma)=\mu\left(\varsigma, \gamma_{0}(\varsigma)\right)+\int_{0}^{\varsigma} \mathcal{K}(\varsigma, \iota, \gamma(\iota)) d \iota
$$

and

$$
\begin{gathered}
\mathscr{S}\left({ }_{0^{+}}^{H C} \mathcal{D}_{\phi(\varsigma)}^{\kappa, \vartheta} \gamma(\varsigma)-{ }_{0^{+}}^{H C} \mathcal{D}_{\phi(\varsigma)}^{\kappa, \vartheta} \gamma_{0}(\varsigma), \vec{\zeta}\right) \circledast \mathscr{M} \mathscr{S}\left(\gamma(\varsigma)-\gamma_{0}(\varsigma), \vec{\zeta}\right) \\
\succeq \psi\left(\varsigma, \frac{\left(1-2 \max \left[\Theta_{1}, \Theta_{2} \Theta_{3}, \Theta_{1} \Theta_{4}, \Theta_{2} \Theta_{3} \Theta_{4}\right]\right) \vec{\zeta}}{\max \left[1, \Theta_{4}\right]}\right),
\end{gathered}
$$

for every $\varsigma \in \mathcal{G}_{1}$ and $\vec{\zeta} \in\left(\mathcal{G}_{2}\right)^{n}$.

Proof We set

$$
\Phi:=\left\{\eta: \mathcal{G}_{1} \rightarrow \mathcal{W}, u \text { is differentiable }\right\}
$$

and introduce the $\mathcal{G}_{4}$-valued metric on $\Phi$ as

$$
\begin{aligned}
\delta\left(\eta, \eta^{\prime}\right) & =\inf \left\{\lambda \in \mathcal{G}_{2}: \mathscr{S}\left({ }_{0^{+}}^{H C} \mathcal{D}_{\phi(\varsigma)}^{\kappa, \vartheta} \eta(\varsigma)-{ }_{0^{+}}^{H C} \mathcal{D}_{\phi(\varsigma)}^{\kappa, \vartheta} \eta^{\prime}(\varsigma), \vec{\zeta}\right) \circledast \mathscr{M} \mathscr{S}\left(\eta(\varsigma)-\eta^{\prime}(\varsigma), \vec{\zeta}\right)\right. \\
& \left.\succeq \psi\left(\varsigma, \frac{\vec{\zeta}}{\lambda}\right), \forall \eta, \eta^{\prime} \in \Phi, \varsigma \in \mathcal{G}_{1}, \vec{\zeta} \in\left(\mathcal{G}_{2}\right)^{n}\right\} .
\end{aligned}
$$

By Theorem 3.1, we have $(\Phi, \delta)$ is a complete $\mathcal{G}_{4}$-valued metric space. 
Now we define the mapping $\Gamma$ from $\Phi$ to $\Phi$ by

$$
\Gamma(\eta(\varsigma))={ }_{0^{+}}^{H} \mathcal{I}_{\phi(\varsigma)}^{\kappa, \vartheta}(\mu(\iota, \eta(\iota)))+{ }_{0^{+}}^{H} \mathcal{I}_{\phi(\varsigma)}^{\kappa, \vartheta}\left(\int_{0}^{\varsigma} \mathcal{K}(\iota, \varpi, \eta(\varpi)) d \varpi\right),
$$

where $\kappa \in \mathcal{G}_{5}^{\circ}, \vartheta \in \mathcal{G}_{5}, \mu: \mathcal{G}_{1} \times \mathcal{W} \rightarrow \mathcal{W}, \mathcal{K}: \mathcal{G}_{1} \times \mathcal{G}_{1} \times \mathcal{W} \rightarrow \mathcal{W}$. We prove $\Gamma$ is a strictly contractive mapping. Let $\eta, \eta^{\prime} \in \Phi, \lambda \in \mathcal{G}_{2}$ and $\delta\left(\eta, \eta^{\prime}\right)<\epsilon$, then we have

$$
\begin{aligned}
& \mathscr{S}\left({ }_{0^{+}}^{H C} \mathcal{D}_{\phi(\varsigma)}^{\kappa, \vartheta} \eta(\varsigma)-{ }_{0^{+}}^{H C} \mathcal{D}_{\phi(\varsigma)}^{\kappa, \vartheta} \eta^{\prime}(\varsigma), \epsilon \vec{\zeta}\right) \circledast \mathscr{M} \mathscr{S}\left(\eta(\varsigma)-\eta^{\prime}(\varsigma), \epsilon \vec{\zeta}\right) \\
& \succeq \psi(\varsigma, \vec{\zeta}), \quad \forall \eta, \eta^{\prime} \in \Phi, \varsigma \in \mathcal{G}_{1}, \vec{\zeta} \in\left(\mathcal{G}_{2}\right)^{n} .
\end{aligned}
$$

Using properties $(\mathcal{S} 2)$ and $(\mathcal{S} 3)$ of Definition 1.4 and (3.8), we have

$$
\begin{aligned}
& \mathscr{S}\left(\left({ }_{0^{+}}^{H C} \mathcal{D}_{\phi(\varsigma)}^{\kappa, \vartheta} \Gamma(\eta(\varsigma))\right)-\left({ }_{0^{+}}^{H C} \mathcal{D}_{\phi(\varsigma)}^{\kappa, \vartheta} \Gamma\left(\eta^{\prime}(\varsigma)\right)\right), 2 \epsilon \vec{\zeta}\right) \\
& \circledast \mathscr{M} \mathscr{S}\left(\Gamma(\eta(\varsigma))-\Gamma\left(\eta^{\prime}(\varsigma)\right), 2 \epsilon \vec{\zeta}\right) \\
& =\mathscr{S}\left(\left[\mu(\varsigma, \eta(\varsigma))-\mu\left(\varsigma, \eta^{\prime}(\varsigma)\right)\right]+\int_{0}^{\varsigma}\left[\mathcal{K}(\varsigma, \iota, \eta(\iota))-\mathcal{K}\left(\varsigma, \iota, \eta^{\prime}(\iota)\right)\right] d \iota, 2 \epsilon \vec{\zeta}\right)
\end{aligned}
$$

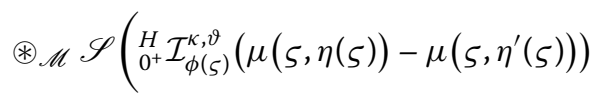

$$
\begin{aligned}
& \left.+{ }_{0^{+}}^{H} \mathcal{I}_{\phi(\varsigma)}^{\kappa, \vartheta}\left(\int_{0}^{s}\left[\mathcal{K}(\varsigma, \iota, \eta(\iota))-\mathcal{K}\left(\varsigma, \iota, \eta^{\prime}(\iota)\right)\right] d \iota\right), 2 \epsilon \vec{\zeta}\right) \\
& \succeq \mathscr{S}\left(\mu(\varsigma, \eta(\varsigma))-\mu\left(\varsigma, \eta^{\prime}(\varsigma)\right), \epsilon \vec{\zeta}\right) \\
& \circledast \mathscr{M} \mathscr{S}\left(\int_{0}^{\varsigma}\left[\mathcal{K}(\varsigma, \iota, \eta(\iota))-\mathcal{K}\left(\varsigma, \iota, \eta^{\prime}(\iota)\right)\right] d \iota, \epsilon \vec{\zeta}\right) \\
& \circledast \mathscr{M} \mathscr{S}\left({ }_{0^{+}}^{H} \mathcal{I}_{\phi(\varsigma)}^{\kappa, \vartheta}\left(\mu(\iota, \eta(\iota))-\mu\left(\iota, \eta^{\prime}(\iota)\right)\right), \epsilon \vec{\zeta}\right)
\end{aligned}
$$

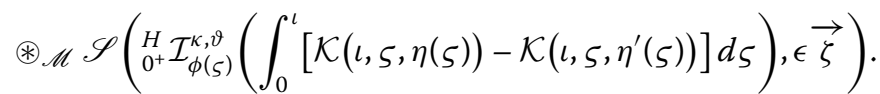

In the last part of (3.9) there are four formulas, in the next steps we work on them to get new formulas derived from the control function $\psi$. Let $0=\Xi_{1}<\Xi_{2}<\cdots<\Xi_{\mathbb{k}_{\mathrm{k}}}=\varsigma$, $\Delta \Xi_{i}=\Xi_{i}-\Xi_{i-1}=\frac{\varsigma}{\mathbb{k}}, i=1,2, \ldots, \mathbb{k}$ and $\|\Delta \Xi\|=\max _{1 \leq i \leq k}\left(\Delta \Xi_{i}\right)$.

Step one. From (3.1) we have

$$
\begin{aligned}
\mathscr{S}\left(\mu(\varsigma, \eta(\varsigma))-\mu\left(\varsigma, \eta^{\prime}(\varsigma)\right), \epsilon \vec{\zeta}\right) & \succeq \mathscr{S}\left(\eta(\varsigma)-\eta^{\prime}(\varsigma), \frac{\epsilon \vec{\zeta}}{\Theta_{1}}\right) \\
& \succeq \psi\left(\varsigma, \frac{\vec{\zeta}}{\Theta_{1}}\right) .
\end{aligned}
$$

Step two. Using $(\mathcal{S} 2)$ and $(\mathcal{S} 3)$ of Definition 1.4, the continuity property of MVF-set $\mathscr{S}$, (3.2) and (3.3), we get

$$
\begin{aligned}
\mathscr{S} & \left(\int_{0}^{\varsigma}\left[\mathcal{K}(\varsigma, \iota, \eta(\iota))-\mathcal{K}\left(\varsigma, \iota, \eta^{\prime}(\iota)\right)\right] d \iota, \epsilon \vec{\zeta}\right) \\
& =\mathscr{S}\left(\lim _{\| \Delta \Xi_{\| \rightarrow 0}} \sum_{j=1}^{\mathbb{k}}\left[\mathcal{K}\left(\varsigma, \Xi_{j}, \eta\left(\Xi_{j}\right)\right)-\mathcal{K}\left(\varsigma, \Xi_{j}, \eta^{\prime}\left(\Xi_{j}\right)\right)\right] \Delta \Xi_{i}, \epsilon \vec{\zeta}\right)
\end{aligned}
$$




$$
\begin{aligned}
& =\lim _{\|\Delta\| \rightarrow 0} \mathscr{S}\left(\sum_{j=1}^{\mathbb{k}}\left[\mathcal{K}\left(\varsigma, \Xi_{j}, \eta\left(\Xi_{j}\right)\right)-\mathcal{K}\left(\varsigma, \Xi_{j}, \nu\left(\Xi_{j}\right)\right)\right] \Delta \Xi_{i}, \epsilon \vec{\zeta}\right) \\
& \geq \lim _{\|\Delta\| \rightarrow 0} \bigwedge_{j=1}^{\mathbb{k}} \mathscr{S}\left(\left[\mathcal{K}\left(\varsigma, \Xi_{j}, \eta\left(\Xi_{j}\right)\right)-\mathcal{K}\left(\varsigma, \Xi_{j}, \eta^{\prime}\left(\Xi_{j}\right)\right)\right] \Delta \Xi_{i}, \frac{\epsilon \vec{\zeta}}{\mathbb{k}}\right) \\
& \succeq \inf _{\partial \in \mathcal{G}_{1}} \mathscr{S}\left(\mathcal{K}(\varsigma, \partial, \eta(ð))-\mathcal{K}\left(\varsigma, \partial, \eta^{\prime}(ð)\right), \frac{\mathbb{k} \epsilon \vec{\zeta}}{\mathbb{k} \xi}\right) \\
& \geq \inf _{\partial \in \mathcal{G}_{1}} \mathscr{S}\left(\eta(ð)-\eta^{\prime}(ð), \frac{\epsilon \vec{\zeta}}{\Theta_{2} \xi}\right) \\
& \geq \inf _{\partial \in \mathcal{G}_{1}} \psi\left(\partial, \frac{\vec{\zeta}}{\Theta_{2} \xi}\right) \\
& \geq \psi\left(\varsigma, \frac{\vec{\zeta}}{\Theta_{2} \Theta_{3}}\right) .
\end{aligned}
$$

Step three. Using (3.4) and (3.10), we get

$$
\mathscr{S}\left({ }_{0^{+}}^{H} \mathcal{I}_{\phi(\varsigma)}^{\kappa, \vartheta}\left(\mu(\iota, \eta(\iota))-\mu\left(\iota, \eta^{\prime}(\iota)\right), \epsilon \vec{\zeta}\right) \succeq \psi\left(\varsigma, \frac{\vec{\zeta}}{\Theta_{1} \Theta_{4}}\right) .\right.
$$

Step four. Using (3.4) and (3.11), we get

$$
\begin{aligned}
& \mathscr{S}\left(\int_{0^{+}}^{H} \mathcal{I}_{\varsigma}^{\kappa, \vartheta}\left(\int_{0}^{\iota}\left[\mathcal{K}(\iota, \varpi, \eta(\varpi))-\mathcal{K}\left(\iota, \varpi, \eta^{\prime}(\varpi)\right)\right] d \varpi\right), \epsilon \vec{\zeta}\right) \\
& \quad \succeq \psi\left(\varsigma, \frac{\vec{\zeta}}{\Theta_{2} \Theta_{3} \Theta_{4}}\right) .
\end{aligned}
$$

Form (3.9), (3.10), (3.11), (3.12) and (3.13), we have

$$
\begin{aligned}
& \mathscr{S}\left({ }_{0^{+}}^{H C} \mathcal{D}_{\phi(\varsigma)}^{\kappa, \vartheta} \Gamma(\eta(\varsigma))-{ }_{0^{+}}^{H C} \mathcal{D}_{\phi(\zeta)}^{\kappa, \vartheta} \Gamma\left(\eta^{\prime}(\varsigma)\right), 2 \epsilon \vec{\zeta}\right) \\
& \circledast \mathscr{M} \mathscr{S}\left(\Gamma(\eta(\varsigma))-\Gamma\left(\eta^{\prime}(\varsigma)\right), 2 \epsilon \vec{\zeta}\right) \\
& \succeq \psi\left(\varsigma, \frac{\vec{\zeta}}{\Theta_{1}}\right) \circledast \mathscr{M} \psi\left(\varsigma, \frac{\vec{\zeta}}{\Theta_{2} \Theta_{3}}\right) \circledast \mathscr{M} \psi\left(\varsigma, \frac{\vec{\zeta}}{\Theta_{1} \Theta_{4}}\right) \circledast \mathscr{M} \psi\left(\varsigma, \frac{\vec{\zeta}}{\Theta_{2} \Theta_{3} \Theta_{4}}\right) \\
& \succeq \psi\left(\varsigma, \frac{\vec{\zeta}}{\max \left[\Theta_{1}, \Theta_{2} \Theta_{3}, \Theta_{1} \Theta_{4}, \Theta_{2} \Theta_{3} \Theta_{4}\right]}\right),
\end{aligned}
$$

and so

$$
\begin{gathered}
\mathscr{S}\left({ }_{0^{+}}^{H C} \mathcal{D}_{\phi(\varsigma)}^{\kappa, \vartheta} \Gamma(\eta(\varsigma))-{ }_{0^{+}}^{H C} \mathcal{D}_{\phi(\varsigma)}^{\kappa, \vartheta} \Gamma\left(\eta^{\prime}(\varsigma)\right), \epsilon \vec{\zeta}\right) \\
\circledast \mathscr{M} \mathscr{S}\left(\Gamma(\eta(\varsigma))-\Gamma\left(\eta^{\prime}(\varsigma)\right), \epsilon \vec{\zeta}\right) \\
\succeq \psi\left(\varsigma, \frac{\vec{\zeta}}{2 \max \left[\Theta_{1}, \Theta_{2} \Theta_{3}, \Theta_{1} \Theta_{4}, \Theta_{2} \Theta_{3} \Theta_{4}\right]}\right),
\end{gathered}
$$


which implies that

$$
\delta\left(\Gamma(\eta), \Gamma\left(\eta^{\prime}\right)\right) \leq 2 \max \left[\Theta_{1}, \Theta_{2} \Theta_{3}, \Theta_{1} \Theta_{4}, \Theta_{2} \Theta_{3} \Theta_{4}\right] \epsilon,
$$

and so

$$
\delta\left(\Gamma(\eta), \Gamma\left(\eta^{\prime}\right)\right) \leq 2 \max \left[\Theta_{1}, \Theta_{2} \Theta_{3}, \Theta_{1} \Theta_{4}, \Theta_{2} \Theta_{3} \Theta_{4}\right] \delta\left(\eta, \eta^{\prime}\right) .
$$

Then $\Gamma$ is a strictly contractive mapping with the Lipschitz constant $2 \max \left[\Theta_{1}, \Theta_{2} \Theta_{3}\right.$, $\left.\Theta_{1} \Theta_{4}, \Theta_{2} \Theta_{3} \Theta_{4}\right]$.

Let $\gamma \in \Phi$, we show that $\delta(\Gamma(\gamma), \gamma)<\infty$. Using (3.4) and (3.5) we get

$$
\begin{aligned}
& \mathscr{S}\left({ }_{0^{+}}^{H C} \mathcal{D}_{\phi(\varsigma)}^{\kappa, \vartheta}[\Gamma(\gamma(\varsigma))-\gamma(\varsigma)], \vec{\zeta}\right) \circledast \mathscr{M} \mathscr{S}(\Gamma(\gamma(\varsigma))-\gamma(\varsigma), \vec{\zeta}) \\
&= \mathscr{S}\left(\mu(\varsigma, \gamma(\varsigma))+\int_{0}^{\varsigma} \mathcal{K}(\varsigma, \iota, \gamma(\iota)) d \iota-{ }_{0^{+}}^{H C} \mathcal{D}_{\phi(\varsigma)}^{\kappa, \vartheta} \gamma(\varsigma), \vec{\zeta}\right) \\
& \circledast \mathscr{M} \mathscr{S}\left({ }_{0^{+}}^{H} \mathcal{I}_{\phi(\zeta)}^{\kappa, \vartheta}(\mu(\iota, \gamma(\iota)))+{ }_{0^{+}}^{H} \mathcal{I}_{\phi(\varsigma)}^{\kappa, \vartheta}\left(\int_{0}^{\iota} \mathcal{K}(\iota, \varpi, \gamma(\varpi)) d \varpi\right)\right. \\
&\left.-{ }_{0^{+}}^{H} \mathcal{I}_{\phi(\varsigma) 0^{+}}^{\kappa, \vartheta} \mathcal{D}_{\phi(\iota)}^{\kappa, \vartheta} \gamma(\iota), \vec{\zeta}\right) \\
&= \mathscr{S}\left(\mu(\varsigma, \gamma(\varsigma))+\int_{0}^{\varsigma} \mathcal{K}(\varsigma, \iota, \gamma(\iota)) d \iota-{ }_{0^{+}}^{H C} \mathcal{D}_{\phi(\varsigma)}^{\kappa, \vartheta} \gamma(\varsigma), \vec{\zeta}\right) \\
& \circledast \mathscr{M} \mathscr{S}\left(\begin{array}{c}
H \\
0^{+}
\end{array} \mathcal{I}_{\phi(\varsigma)}^{\kappa, \vartheta}\left[\mu(\iota, \gamma(\iota))+\int_{0}^{\iota} \mathcal{K}(\iota, \varpi, \gamma(\varpi)) d \varpi-{ }_{0^{+}}^{H C} \mathcal{D}_{\phi(\varsigma)}^{\kappa, \vartheta} \gamma(\iota)\right], \vec{\zeta}\right) \\
& \succeq \psi(\varsigma, \vec{\zeta}) \circledast \mathscr{M} \psi\left(\varsigma, \frac{\vec{\zeta}}{\Theta_{4}}\right) \\
& \succeq \psi\left(\varsigma, \frac{\vec{\zeta}}{\max \left[1, \Theta_{4}\right]}\right),
\end{aligned}
$$

for every $\vec{\zeta} \in\left(\mathcal{G}_{2}\right)^{n}$. Then we have $\delta(\Gamma(\gamma), \gamma)<\max \left[1, \Theta_{4}\right]<\infty$.

Now Theorem 1.5 enables us to find an element $\gamma_{0}$ in $\Phi$ satisfying the following:

(1) $\gamma_{0}$ is a fixed point of $\Gamma$, i.e.,

$$
\begin{aligned}
\gamma_{0}(\varsigma) & =\Gamma\left(\gamma_{0}(\varsigma)\right) \\
& ={ }_{0^{+}}^{H} \mathcal{I}_{\phi(\varsigma)}^{\kappa, \vartheta}\left(\mu\left(\iota, \gamma_{0}(\iota)\right)\right)+{ }_{0^{+}}^{H} \mathcal{I}_{\phi(\varsigma)}^{\kappa, \vartheta}\left(\int_{0}^{\iota} \mathcal{K}\left(\iota, \varpi, \gamma_{0}(\varpi)\right) d \varpi\right),
\end{aligned}
$$

which is unique in the set

$$
\Phi^{*}=\{\eta \in \Phi: \delta(\Gamma(\gamma), \eta)<\infty\} .
$$

Taking ${ }_{0^{+}}^{H C} \mathcal{D}_{\phi(\varsigma)}^{\kappa, \vartheta}$ from (3.19) we get

$$
{ }_{0^{+}}^{H C} \mathcal{D}_{\phi(\varsigma)}^{\kappa, \vartheta} \gamma_{0}(\varsigma)=\mu\left(\varsigma, \gamma_{0}(\varsigma)\right)+\int_{0}^{\varsigma} \mathcal{K}\left(\varsigma, \iota, \gamma_{0}(\iota)\right) d \iota,
$$


where $\kappa \in \mathcal{G}_{5}^{\circ}, \vartheta \in \mathcal{G}_{5}, \mu: \mathcal{G}_{1} \times \mathcal{W} \rightarrow \mathcal{W}, \mathcal{K}: \mathcal{G}_{1} \times \mathcal{G}_{1} \times \mathcal{W} \rightarrow \mathcal{W}$.

(2) $\delta\left(\Gamma^{\tau}(\gamma), \gamma_{0}\right) \rightarrow 0$ as $\tau \rightarrow \infty$.

(3) $\delta\left(\gamma, \gamma_{0}\right) \leq \frac{1}{1-2 \max \left[\Theta_{1}, \Theta_{2} \Theta_{3}, \Theta_{1} \Theta_{4}, \Theta_{2} \Theta_{3} \Theta_{4}\right]} \delta(\Gamma(\gamma), \gamma) \leq \frac{\max \left[1, \Theta_{4}\right]}{1-2 \max \left[\Theta_{1}, \Theta_{2} \Theta_{3}, \Theta_{1} \Theta_{4}, \Theta_{2} \Theta_{3} \Theta_{4}\right]}$, which implies that

$$
\begin{gathered}
\left.\mathscr{S}{ }_{0^{+}}^{H C} \mathcal{D}_{\phi(\varsigma)}^{\kappa, \vartheta} \gamma(\varsigma){ }_{0^{+}}^{H C} \mathcal{D}_{\phi(\varsigma)}^{\kappa, \vartheta} \gamma_{0}(\varsigma), \vec{\zeta}\right) \circledast \mathscr{M} \mathscr{S}\left(\gamma(\varsigma)-\gamma_{0}(\varsigma), \vec{\zeta}\right) \\
\succeq \psi\left(\varsigma, \frac{\left(1-2 \max \left[\Theta_{1}, \Theta_{2} \Theta_{3}, \Theta_{1} \Theta_{4}, \Theta_{2} \Theta_{3} \Theta_{4}\right]\right) \vec{\zeta}}{\max \left[1, \Theta_{4}\right]}\right),
\end{gathered}
$$

for every $\varsigma \in \mathcal{G}_{1}$ and $\vec{\zeta} \in\left(\mathcal{G}_{2}\right)^{n}$.

Now we show that the fixed point in $\Phi^{*}$ is unique. Let $\gamma_{0}^{\prime}$ be an element of $\Phi$ satisfying (3.6) and (3.7), we prove that $\gamma_{0}^{\prime}=\gamma_{0}$ and $\gamma_{0}^{\prime} \in \Phi^{*}$. From (3.6) we get

$$
{ }_{0^{+}}^{H C} \mathcal{D}_{\phi(\varsigma)}^{\kappa, \vartheta} \gamma_{0}^{\prime}(\varsigma)=\mu\left(\varsigma, \gamma_{0}^{\prime}(\varsigma)\right)+\int_{0}^{\varsigma} \mathcal{K}\left(\varsigma, \iota, \gamma_{0}^{\prime}(\iota)\right) d \iota,
$$

and so

$$
\gamma_{0}^{\prime}(\varsigma)={ }_{0^{+}}^{H} \mathcal{I}_{\phi(\varsigma)}^{\kappa, \vartheta} \mu\left(\iota, \gamma_{0}^{\prime}(\iota)\right)+{ }_{0^{+}}^{H} \mathcal{I}_{\phi(\varsigma)}^{\kappa, \vartheta} \int_{0}^{\iota} \mathcal{K}\left(\iota, \varpi, \gamma_{0}^{\prime}(\varpi)\right) d \varpi=\Gamma\left(\gamma_{0}^{\prime}(\varsigma)\right),
$$

where $\kappa \in \mathcal{G}_{5}^{\circ}, \vartheta \in \mathcal{G}_{5}, \mu: \mathcal{G}_{1} \times \mathcal{W} \rightarrow \mathcal{W}, \mathcal{K}: \mathcal{G}_{1} \times \mathcal{G}_{1} \times \mathcal{W} \rightarrow \mathcal{W}$.

Now we show that

$$
\gamma_{0}^{\prime} \in\{\eta \in \Phi: \delta(\Gamma(\gamma), \eta)<\infty\}
$$

i.e., $\delta\left(\Gamma(\gamma), \gamma_{0}^{\prime}\right)<\infty$. We set $\theta=\frac{1-2 \max \left[\Theta_{1}, \Theta_{2} \Theta_{3}, \Theta_{1} \Theta_{4}, \Theta_{2} \Theta_{3} \Theta_{4}\right]}{\max \left[1, \Theta_{4}\right]}$, from (3.7) we get

$$
\begin{aligned}
& \mathscr{S}\left(\begin{array}{l}
H C \\
0^{+}
\end{array} \mathcal{D}_{\phi(\varsigma)}^{\kappa, \vartheta} \gamma(\varsigma)-{ }_{0^{+}}^{H C} \mathcal{D}_{\phi(\varsigma)}^{\kappa, \vartheta} \gamma_{0}^{\prime}(\varsigma), \vec{\zeta}\right) \circledast \mathscr{M} \mathscr{S}\left(\gamma(\varsigma)-\gamma_{0}^{\prime}(\varsigma), \vec{\zeta}\right) \\
& \quad \succeq \psi(\varsigma, \theta \vec{\zeta}),
\end{aligned}
$$

for every $\varsigma \in \mathcal{G}_{1}$ and $\vec{\zeta} \in\left(\mathcal{G}_{2}\right)^{n}$.

From (3.1) and (3.23) we get

$$
\begin{aligned}
\mathscr{S}\left(\mu(\varsigma, \gamma(\varsigma))-\mu\left(\varsigma, \gamma_{0}^{\prime}(\varsigma)\right), \vec{\zeta}\right) & \succeq \mathscr{S}\left(\gamma(\varsigma)-\gamma_{0}^{\prime}(\varsigma), \frac{\vec{\zeta}}{\Theta_{1}}\right) \\
& \succeq \psi\left(\varsigma, \theta \frac{\vec{\zeta}}{\Theta_{1}}\right),
\end{aligned}
$$

also, from (3.2) and (3.23) we get

$$
\begin{aligned}
\mathscr{S}\left(\mathcal{K}(\varsigma, \iota, \gamma(\iota))-\mathcal{K}\left(\varsigma, \iota, \gamma_{0}^{\prime}(\iota)\right), \vec{\zeta}\right) & \geq \mathscr{S}\left(\gamma(\iota)-\gamma_{0}^{\prime}(\iota), \frac{\vec{\zeta}}{\Theta_{2}}\right) \\
& \geq \psi\left(\varsigma, \theta \frac{\vec{\zeta}}{\Theta_{2}}\right),
\end{aligned}
$$


for every $\varsigma \in \mathcal{G}_{1}, \iota \leq \varsigma$ and $\vec{\zeta} \in\left(\mathcal{G}_{2}\right)^{n}$. Now, using step two and (3.25), we get

$$
\begin{aligned}
\mathscr{S}\left(\int_{0}^{\varsigma}\left[\mathcal{K}(\varsigma, \iota, \gamma(\iota))-\mathcal{K}\left(\varsigma, \iota, \gamma_{0}^{\prime}(\iota)\right)\right] d \iota, \vec{\zeta}\right) & \succeq \psi\left(\varsigma, \frac{\vec{\zeta}}{\Theta_{2} \Theta_{3}}\right) \\
& \geq \psi\left(\varsigma, \theta \frac{\vec{\zeta}}{\Theta_{2} \Theta_{3}}\right) .
\end{aligned}
$$

Using the triangular inequality $(\mathcal{S} 3),(3.24)$ and (3.26) we get

$$
\begin{aligned}
& \mathscr{S}\left(\mu(\varsigma, \gamma(\varsigma))-\mu\left(\varsigma, \gamma_{0}^{\prime}(\varsigma)\right)+\int_{0}^{\varsigma}\left[\mathcal{K}(\varsigma, \iota, \gamma(\iota))-\mathcal{K}\left(\varsigma, \iota, \gamma_{0}^{\prime}(\iota)\right)\right] d \iota, 2 \vec{\zeta}\right) \\
& \succeq \mathscr{S}\left(\mu(\varsigma, \gamma(\varsigma))-\mu\left(\varsigma, \gamma_{0}^{\prime}(\varsigma)\right), \vec{\zeta}\right) \\
& \circledast \mathscr{M} \mathscr{S}\left(\int_{0}^{\varsigma}\left[\mathcal{K}(\varsigma, \iota, \gamma(\iota))-\mathcal{K}\left(\varsigma, \iota, \gamma_{0}^{\prime}(\iota)\right)\right] d \iota, \vec{\zeta}\right) \\
& \geq \psi\left(\varsigma, \theta \frac{\vec{\zeta}}{\Theta_{1}}\right) \circledast \mathscr{M} \psi\left(\varsigma, \theta \frac{\vec{\zeta}}{\Theta_{2} \Theta_{3}}\right) \\
& \geq \psi\left(\varsigma, \theta \frac{\vec{\zeta}}{\max \left[\Theta_{1}, \Theta_{2} \Theta_{3}\right]}\right),
\end{aligned}
$$

and so

$$
\begin{aligned}
& \mathscr{S}\left(\mu(\varsigma, \gamma(\varsigma))-\mu\left(\varsigma, \gamma_{0}^{\prime}(\varsigma)\right)+\int_{0}^{\varsigma}\left[\mathcal{K}(\varsigma, \iota, \gamma(\iota))-\mathcal{K}\left(\varsigma, \iota, \gamma_{0}^{\prime}(\iota)\right)\right] d \iota, \vec{\zeta}\right) \\
& \quad \succeq \psi\left(\varsigma, \theta \frac{\vec{\zeta}}{2 \max \left[\Theta_{1}, \Theta_{2} \Theta_{3}\right]}\right) .
\end{aligned}
$$

We apply (3.4) to get

$$
\begin{aligned}
& \mathscr{S}\left({ }_{0^{+}} \mathcal{I}_{\phi(\varsigma)}^{\kappa, \vartheta}\left[\mu(\iota, \gamma(\iota))-\mu\left(\iota, \gamma_{0}^{\prime}(\iota)\right)\right]\right. \\
& \left.\quad+{ }_{0^{+}}^{H} \mathcal{I}_{\phi(\zeta)}^{\kappa, \vartheta}\left(\int_{0}^{\iota}\left[\mathcal{K}(\iota, \varpi, \gamma(\varpi))-\mathcal{K}\left(\iota, \varpi, \gamma_{0}^{\prime}(\varpi)\right)\right] d \varpi\right), \vec{\zeta}\right) \\
& \succeq \psi\left(\varsigma, \theta \frac{\vec{\zeta}}{2 \Theta_{4} \max \left[\Theta_{1}, \Theta_{2} \Theta_{3}\right]}\right),
\end{aligned}
$$

for every $\varsigma \in \mathcal{G}_{1}, \iota \leq \varsigma$ and $\vec{\zeta} \in\left(\mathcal{G}_{2}\right)^{n}$.

Using (3.28) and (3.29) we get

$$
\begin{aligned}
& \mathscr{S}\left({ }_{0^{+}}^{H C} \mathcal{D}_{\phi(\varsigma)}^{\kappa, \vartheta}\left[\Gamma(\gamma(\varsigma))-\gamma_{0}^{\prime}(\varsigma)\right], \vec{\zeta}\right) \circledast \mathscr{M} \mathscr{S}\left(\Gamma(\gamma(\varsigma))-\gamma_{0}^{\prime}(\varsigma), \vec{\zeta}\right) \\
&=\mathscr{S}\left(\mu(\varsigma, \gamma(\varsigma))-\mu\left(\varsigma, \gamma_{0}^{\prime}(\varsigma)\right)+\int_{0}^{\varsigma}\left[\mathcal{K}(\varsigma, \iota, \gamma(\iota))-\mathcal{K}\left(\varsigma, \iota, \gamma_{0}^{\prime}(\iota)\right)\right] d \iota, \vec{\zeta}\right) \\
& \circledast \mathscr{M} \mathscr{S}\left({ }_{0^{+}}^{H} \mathcal{I}_{\phi(\varsigma)}^{\kappa, \vartheta}\right)\left[\mu(\iota, \gamma(\iota))-\mu\left(\iota, \gamma_{0}^{\prime}(\iota)\right)\right]
\end{aligned}
$$




$$
\begin{aligned}
& \left.+{ }_{0^{+}}^{H} \mathcal{I}_{\phi(\varsigma)}^{\kappa, \vartheta}\left(\int_{0}^{\iota}\left[\mathcal{K}(\iota, \varpi, \gamma(\varpi))-\mathcal{K}\left(\iota, \varpi, \gamma_{0}^{\prime}(\varpi)\right)\right] d \varpi\right), \vec{\zeta}\right) \\
\succeq & \psi\left(\varsigma, \theta \frac{\vec{\zeta}}{2 \max \left[\Theta_{1}, \Theta_{2} \Theta_{3}\right]}\right) \circledast \mathscr{M} \psi\left(\varsigma, \theta \frac{\vec{\zeta}}{2 \Theta_{4} \max \left[\Theta_{1}, \Theta_{2} \Theta_{3}\right]}\right) \\
\succeq & \psi\left(\varsigma, \theta \frac{\vec{\zeta}}{2 \max \left[\Theta_{1}, \Theta_{2} \Theta_{3}\right]\left(1+\Theta_{4}\right)}\right),
\end{aligned}
$$

which implies that $\delta\left(\Gamma(\gamma), \gamma_{0}^{\prime}\right) \leq \frac{2 \max \left[\Theta_{1}, \Theta_{2} \Theta_{3}\right]\left(1+\Theta_{4}\right)}{\theta}<\infty$, then $\gamma_{0}^{\prime} \in \Phi^{*}$.

\section{Best approximation of $\phi$-Hadamard fractional Volterra integral equation}

Now we are ready to study UHR stability of the $\phi$-Hadamard fractional Volterra integral equation

$$
\eta(\varsigma)=\mu(\varsigma, \eta(\varsigma))+{ }_{0^{+}}^{H} \mathcal{I}_{\phi(\varsigma)}^{\kappa, \vartheta} \mathcal{K}(\varsigma, \iota, \eta(\iota)),
$$

where $\kappa \in \mathcal{G}_{5}^{\circ}, \vartheta \in \mathcal{G}_{5}, \mu: \mathcal{G}_{1} \times W \rightarrow W, \mathcal{K}: \mathcal{G}_{1} \times \mathcal{G}_{1} \times W \rightarrow W$ and get the best approximation with better estimate for the pseudo- $\phi$-Hadamard fractional Volterra integral equation.

Theorem 4.1 Let $(\mathcal{W}, \mathscr{S}, \circledast)$ be an MVFB-space and $\Theta_{1}, \Theta_{2}, \Theta_{3}, \Theta_{4}$ and $\xi$ be positive constant such that $\max \left[\Theta_{1}, \Theta_{2} \Theta_{3}, \Theta_{1} \Theta_{4}, \Theta_{2} \Theta_{3} \Theta_{4}\right]<0.5$. Assume that the continuous mappings $\mu: \mathcal{G}_{1} \times \mathcal{W} \rightarrow \mathcal{W}, \mathcal{K}: \mathcal{G}_{1} \times \mathcal{G}_{1} \times \mathcal{W} \rightarrow \mathcal{W}$ with MVF-set $\psi: \mathcal{W} \times\left(\mathcal{G}_{2}\right)^{n} \rightarrow$ $\operatorname{diag} \mathcal{M}_{n}\left(\mathcal{G}_{3}\right)$ satisfy (3.1), (3.2), (3.3) and (3.4).

Let $\gamma: \mathcal{G}_{1} \rightarrow \mathcal{W}$ be a differentiable function satisfying

$$
\mathscr{S}\left(\gamma(\varsigma)-\mu(\varsigma, \gamma(\varsigma))-{ }_{0^{+}}^{H} \mathcal{I}_{\phi(\varsigma)}^{\kappa, \vartheta} \mathcal{K}(\varsigma, \iota, w(\iota)), \vec{\zeta}\right) \succeq \psi(\varsigma, \vec{\zeta})
$$

for every $\varsigma \in \mathcal{G}_{1}$ and $\vec{\zeta} \in\left(\mathcal{G}_{2}\right)^{n}$. Then we have a unique differentiable function $\gamma_{0}: \mathcal{G}_{1} \rightarrow W$ such that

$$
\gamma_{0}(\varsigma)=\mu\left(\varsigma, \gamma_{0}(\varsigma)\right)+{ }_{0^{+}}^{H} \mathcal{I}_{\phi(\varsigma)}^{\kappa, \vartheta} \mathcal{K}\left(\varsigma, \iota, \gamma_{0}(\iota)\right)
$$

and

$$
\begin{aligned}
& \mathscr{S}\left(\gamma(\varsigma)-\gamma_{0}(\varsigma), \vec{\zeta}\right) \\
& \quad \succeq \psi\left(\varsigma, \frac{\left(1-2 \max \left[\Theta_{1}, \Theta_{2} \Theta_{3}, \Theta_{1} \Theta_{4}, \Theta_{2} \Theta_{3} \Theta_{4}\right]\right) \vec{\zeta}}{\max \left[1, \Theta_{4}\right]}\right),
\end{aligned}
$$

for every $\varsigma \in \mathcal{G}_{1}$ and $\vec{\zeta} \in\left(\mathcal{G}_{2}\right)^{n}$.

Proof We set

$$
\Phi:=\left\{\eta: \mathcal{G}_{1} \rightarrow \mathcal{W}, \eta \text { is differentiable }\right\}
$$


and introduce the $\mathcal{G}_{4}$-valued metric on $\Phi$ as

$$
\begin{aligned}
& \delta\left(\eta, \eta^{\prime}\right) \\
& \quad=\inf \left\{\lambda \in \mathcal{G}_{2}: \mathscr{S}\left(\eta(\varsigma)-\eta^{\prime}(\varsigma), \vec{\zeta}\right) \geq \psi\left(\varsigma, \frac{\vec{\zeta}}{\lambda}\right), \forall \eta, \eta^{\prime} \in \Phi, \varsigma \in \mathcal{G}_{1}, \vec{\zeta} \in\left(\mathcal{G}_{2}\right)^{n}\right\} .
\end{aligned}
$$

By Theorem 3.1, we have $(\Phi, \delta)$ is a complete $\mathcal{G}_{4}$-valued metric space.

Now we define the mapping $\Gamma$ from $\Phi$ to $\Phi$ by

$$
\Gamma(\eta(\varsigma))=\mu(\varsigma, \eta(\varsigma))+{ }_{0^{+}}^{H} \mathcal{I}_{\phi(\varsigma)}^{\kappa, \vartheta}(\mathcal{K}(\varsigma, \iota, \eta(\iota)))
$$

where $\kappa \in \mathcal{G}_{5}^{\circ}, \vartheta \in \mathcal{G}_{5}, \mu: \mathcal{G}_{1} \times \mathcal{W} \rightarrow \mathcal{W}, \mathcal{K}: \mathcal{G}_{1} \times \mathcal{G}_{1} \times \mathcal{W} \rightarrow \mathcal{W}$. We prove $\Gamma$ is a strictly contractive mapping. Let $\eta, \eta^{\prime} \in \Phi, \lambda \in \mathcal{G}_{2}$ and $\delta\left(\eta, \eta^{\prime}\right)<\epsilon$, then we have

$$
\mathscr{S}\left(\eta(\varsigma)-\eta^{\prime}(\varsigma), \epsilon \vec{\zeta}\right) \geq \psi(\varsigma, \vec{\zeta}), \quad \forall \eta, \eta^{\prime} \in \Phi, \varsigma \in \mathcal{G}_{1}, \vec{\zeta} \in\left(\mathcal{G}_{2}\right)^{n}
$$

Using properties $(\mathcal{S} 2)$ and $(\mathcal{S} 3)$ of Definition 1.4, (3.1), (3.2), (3.3), (3.4) and (4.5), we have

$$
\begin{aligned}
\mathscr{S} & \left(\Gamma(\eta(\varsigma))-\Gamma\left(\eta^{\prime}(\varsigma)\right), 2 \epsilon \vec{\zeta}\right) \\
= & \mathscr{S}\left(\left[\mu(\varsigma, \eta(\varsigma))-\mu\left(\varsigma, \eta^{\prime}(\varsigma)\right)\right]\right. \\
& \left.+{ }_{0^{+}}^{H} \mathcal{I}_{\phi(\varsigma)}^{\kappa, \vartheta}\left[\mathcal{K}(\varsigma, \iota, \eta(\iota))-\mathcal{K}\left(\varsigma, \iota, \eta^{\prime}(\iota)\right)\right], 2 \epsilon \vec{\zeta}\right) \\
\succeq & \mathscr{S}\left(\mu(\varsigma, \eta(\varsigma))-\mu\left(\varsigma, \eta^{\prime}(\varsigma)\right), \epsilon \vec{\zeta}\right) \circledast \mathscr{M} \mathscr{S}\left(\mathcal{K}(\varsigma, \iota, \eta(\iota))-\mathcal{K}\left(\varsigma, \iota, \eta^{\prime}(\iota)\right), \epsilon \vec{\zeta}\right) \\
\succeq & \psi\left(\varsigma, \frac{\vec{\zeta}}{\Theta_{1}}\right) \circledast \mathscr{M} \psi\left(\varsigma, \frac{\vec{\zeta}}{\Theta_{2} \Theta_{4}}\right) \\
\geq & \psi\left(\varsigma, \frac{\vec{\zeta}}{\max \left[\Theta_{1}, \Theta_{2} \Theta_{4}\right]}\right),
\end{aligned}
$$

and so

$$
\mathscr{S}\left(\Gamma(\eta(\varsigma))-\Gamma\left(\eta^{\prime}(\varsigma)\right), \epsilon \vec{\zeta}\right) \geq \psi\left(\varsigma, \frac{\vec{\zeta}}{2 \max \left[\Theta_{1}, \Theta_{2} \Theta_{4}\right]}\right),
$$

for every $\varsigma \in \mathcal{G}_{1}$ and $\vec{\zeta} \in\left(\mathcal{G}_{2}\right)^{n}$. Then

$$
\delta\left(\Gamma(\eta), \Gamma\left(\eta^{\prime}\right)\right) \leq 2 \max \left[\Theta_{1}, \Theta_{2} \Theta_{4}\right] \epsilon,
$$

and so

$$
\delta\left(\Gamma(\eta), \Gamma\left(\eta^{\prime}\right)\right) \leq 2 \max \left[\Theta_{1}, \Theta_{2} \Theta_{4}\right] \delta\left(\eta, \eta^{\prime}\right) .
$$

Then $\Gamma$ is a strictly contractive function with the Lipschitz constant $2 \max \left[\Theta_{1}, \Theta_{2} \Theta_{4}\right]$. 
Let $\gamma \in \Phi$. Then we show that $\delta(\Gamma(\gamma), \gamma)<\infty$. Using (4.2) we get

$$
\begin{aligned}
\mathscr{S}(\Gamma(\gamma(\varsigma))-\gamma(\varsigma), \vec{\zeta}) & =\mathscr{S}\left(\mu(\varsigma, \gamma(\varsigma))+{ }_{0^{+}}^{H} \mathcal{I}_{\phi(\zeta)}^{\kappa, \vartheta} \mathcal{K}(\varsigma, \iota, \gamma(\iota))-\gamma(\varsigma), \vec{\zeta}\right) \\
& \succeq \psi(\varsigma, \vec{\zeta})
\end{aligned}
$$

for every $\vec{\zeta} \in\left(\mathcal{G}_{2}\right)^{n}$. Then we have $\delta(\Gamma(\gamma), \gamma)<1$

Now Theorem 1.5 enables us to find an element $\gamma_{0}$ in $\Phi$ satisfying the following:

(1) $\gamma_{0}$ is a fixed point of $\Gamma$, i.e.,

$$
\begin{aligned}
\gamma_{0}(\varsigma) & =\Gamma\left(\gamma_{0}(\varsigma)\right) \\
& =\mu\left(\varsigma, \gamma_{0}(\varsigma)\right)+{ }_{0^{+}}^{H} \mathcal{I}_{\phi(\varsigma)}^{\kappa, \vartheta}\left(\mathcal{K}\left(\varsigma, \iota, \gamma_{0}(\iota)\right)\right),
\end{aligned}
$$

which is unique in the set

$$
\Phi^{*}=\{\eta \in \Phi: \delta(\Gamma(\gamma), \eta)<\infty\} .
$$

(2) $\delta\left(\Gamma^{\tau}(\gamma), \gamma_{0}\right) \rightarrow 0$ as $\tau \rightarrow \infty$;

(3) $\delta\left(\gamma, \gamma_{0}\right) \leq \frac{1}{1-2 \max \left[\Theta_{1}, \Theta_{2} \Theta_{4}\right]} \delta(\Gamma(\gamma), \gamma) \leq \frac{1}{1-2 \max \left[\Theta_{1}, \Theta_{2} \Theta_{4}\right]}$, which implies that

$$
\mathscr{S}\left(\gamma(\varsigma)-\gamma_{0}(\varsigma), \vec{\zeta}\right) \succeq \psi\left(\varsigma,\left(1-2 \max \left[\Theta_{1}, \Theta_{2} \Theta_{4}\right]\right) \vec{\zeta}\right)
$$

for every $\varsigma \in \mathcal{G}_{1}$ and $\vec{\zeta} \in\left(\mathcal{G}_{2}\right)^{n}$.

\section{Application}

In this section, we apply the main results to solving some examples.

Example 5.1 Let $(\mathbb{R}, \mathscr{S}, \circledast)$ be an MVFB-space. Consider $\eta, \eta^{\prime}: \mathcal{G}_{1} \rightarrow \mathbb{R}$ and define $\mu(\varsigma, \eta(\varsigma))=\ln \sqrt{|\eta(\varsigma)|}$. Let $\mathbf{E}_{c, d}$ be the two-parameter Mittag-Leffler function in which $\Re(c)>0$ and $\Re(d)>0$, define $\mathcal{K}: \mathcal{G}_{1} \times \mathcal{G}_{1} \times \mathbb{R} \rightarrow \mathbb{R}$ as $\mathcal{K}(\varsigma, \iota, \eta(\iota))=\mathbf{E}_{c, d}(\varsigma-\iota) \eta(\iota)$ for every $\varsigma \in \mathcal{G}_{1}$ and $\iota \leq \varsigma$.

Then we have

$$
\begin{aligned}
\mathscr{S}\left(\mathcal{K}(\varsigma, \iota, \eta(\iota))-\mathcal{K}\left(\varsigma, \iota, \eta^{\prime}(\iota)\right), \vec{\zeta}\right) & =\mathscr{S}\left(\mathbf{E}_{c, d}(\varsigma-\iota)\left[\eta(\iota)-\eta^{\prime}(\iota)\right], \vec{\zeta}\right) \\
& \succeq \mathscr{S}\left(\eta(\iota)-\eta^{\prime}(\iota), \frac{\vec{\zeta}}{\left|\mathbf{E}_{c, d}(\varsigma-\iota)\right|}\right) \\
& \succeq \mathscr{S}\left(\eta(\iota)-\eta^{\prime}(\iota), \frac{\vec{\zeta}}{\Lambda}\right), \\
\mathscr{S}\left(\mu(\varsigma, \eta(\varsigma))-\mu\left(\varsigma, \eta^{\prime}(\varsigma)\right), \vec{\zeta}\right) & =\mathscr{S}\left(\ln \sqrt{|\eta(\varsigma)|}-\ln \sqrt{\left|\eta^{\prime}(\varsigma)\right|,} \vec{\zeta}\right) \\
& =\mathscr{S}\left(\ln \frac{\sqrt{|\eta(\varsigma)|}}{\sqrt{\left|\eta^{\prime}(\varsigma)\right|}}, \vec{\zeta}\right) \\
& \succeq \mathscr{S}\left(\ln \left(1+\left(\frac{\sqrt{|\eta(\varsigma)|}}{\sqrt{\left|\eta^{\prime}(\varsigma)\right|}}-1\right)\right), \vec{\zeta}\right)
\end{aligned}
$$




$$
\begin{aligned}
& \succeq \mathscr{S}\left(\frac{\sqrt{|\eta(\varsigma)|}}{\sqrt{\left|\eta^{\prime}(\varsigma)\right|}}-1, \vec{\zeta}\right) \\
& \succeq \mathscr{S}\left(\frac{\sqrt{|\eta(\varsigma)|}-\sqrt{\left|\eta^{\prime}(\varsigma)\right|}}{\sqrt{\min \left(\left|\eta^{\prime}(\varsigma)\right|\right)}}, \vec{\zeta}\right) \\
& \succeq \mathscr{S}\left(\sqrt{|\eta(\varsigma)|}-\sqrt{\left|\eta^{\prime}(\varsigma)\right|}, \vec{\zeta}\right) \\
& \succeq \mathscr{S}\left(\frac{|\eta(\varsigma)|-\left|\eta^{\prime}(\varsigma)\right|}{\sqrt{|\eta(\varsigma)|}+\sqrt{\left|\eta^{\prime}(\varsigma)\right|}}, \vec{\zeta}\right) \\
& \succeq \mathscr{S}\left(\frac{\left|\eta(\varsigma)-\eta^{\prime}(\varsigma)\right|}{\sqrt{\max \left(|\eta(\varsigma)|,\left|\eta^{\prime}(\varsigma)\right|\right)}}, \vec{\zeta}\right) \\
& \succeq \mathscr{S}\left(\complement\left(\eta(\varsigma)-\eta^{\prime}(\varsigma)\right), \vec{\zeta}\right) \\
& =\mathscr{S}\left(\eta(\varsigma)-\eta^{\prime}(\varsigma), \frac{\vec{\zeta}}{\complement}\right) \text {, }
\end{aligned}
$$

for some $\Lambda \in \mathcal{G}_{2}$ and $\complement \in \mathcal{G}_{3}$. Consider the MVF-set $\psi: \mathcal{G}_{1} \times\left(\mathcal{G}_{2}\right)^{4} \rightarrow \operatorname{diag} \mathcal{M}_{4}\left(\mathcal{G}_{3}\right)$, that is defined as follows:

$$
\psi(\varsigma, \vec{\zeta})=\operatorname{diag}\left[\mathbf{E}_{\aleph}\left(-\frac{|\zeta|}{\zeta_{1}}\right), \frac{\zeta_{2}}{\zeta_{2}+|\zeta|}, \exp \left(-\frac{|\zeta|}{\zeta_{3}}\right), \mathbf{E}_{\aleph}\left(-\frac{|\zeta|}{\zeta_{4}}\right)\right]
$$

for every $\varsigma \in \mathcal{G}_{1}, \vec{\zeta} \in\left(\mathcal{G}_{2}\right)^{4}$ and $\aleph \in \mathcal{G}_{3}$.

Let $\gamma: \mathcal{G}_{1} \rightarrow \mathbb{R}$ be a differentiable function satisfying

$$
\begin{aligned}
& \mathscr{S}\left({ }_{0^{+}}^{H C} \mathcal{D}_{\phi(\varsigma)}^{\kappa, \vartheta} \gamma(\varsigma)-\ln \sqrt{|\gamma(\varsigma)|}-\int_{0}^{\varsigma} \mathbf{E}_{c, d}(\varsigma-\iota) \gamma(\iota) d \iota, \vec{\zeta}\right) \\
& \succeq \psi(\varsigma, \vec{\zeta})
\end{aligned}
$$

for every $\varsigma \in \mathcal{G}_{1}$ and $\vec{\zeta} \in\left(\mathcal{G}_{2}\right)^{4}$. Now Theorem 3.2 implies that, if $\max \left[\mathcal{C}, \Lambda \Theta_{3}, \complement \Theta_{4}\right.$, $\left.\Lambda \Theta_{3} \Theta_{4}\right]<0.5$, we can find a unique differentiable function $\gamma_{0}: \mathcal{G}_{1} \rightarrow \mathbb{R}$ such that

$$
{ }_{0^{+}}^{H C} \mathcal{D}_{\phi(\varsigma)}^{\kappa, \vartheta} \gamma_{0}(\varsigma)=\ln \sqrt{\left|\gamma_{0}(\varsigma)\right|}+\int_{0}^{\varsigma} \mathbf{E}_{c, d}(\varsigma-\iota) \gamma_{0}(\iota) d \iota
$$

and

$$
\begin{aligned}
& \mathscr{S}\left({ }_{0^{+}}^{H C} \mathcal{D}_{\phi(\varsigma)}^{\kappa, \vartheta} \gamma(\varsigma)-{ }_{0^{+}}^{H C} \mathcal{D}_{\phi(\varsigma)}^{\kappa, \vartheta} \gamma_{0}(\varsigma), \vec{\zeta}\right) \circledast \mathscr{M} \mathscr{S}\left(\gamma(\varsigma)-\gamma_{0}(\varsigma), \vec{\zeta}\right) \\
& \succeq \psi\left(\varsigma, \frac{\left(1-2 \max \left[\complement, \Lambda \Theta_{3}, \complement \Theta_{4}, \Lambda \Theta_{3} \Theta_{4}\right]\right) \vec{\zeta}}{\max \left[1, \Theta_{4}\right]}\right),
\end{aligned}
$$

for every $\varsigma \in \mathcal{G}_{1}$ and $\vec{\zeta} \in\left(\mathcal{G}_{2}\right)^{4}$.

Example 5.2 Let $(\mathbb{R}, \mathscr{S}, \circledast)$ be an MVFB-space. Consider $\eta, \eta^{\prime}: \mathcal{G}_{1} \rightarrow \mathbb{R}$ and define $\mu(\varsigma, \eta(\varsigma))=\ln \sqrt{|\eta(\varsigma)|}$. Let $\mathbf{E}_{c, d}$ be the two-parameter Mittag-Leffler function, in which $\Re(c)>0$ and $\Re(d)>0$, define $\mathcal{K}: \mathcal{G}_{1} \times \mathcal{G}_{1} \times \mathbb{R} \rightarrow \mathbb{R}$ as $\mathcal{K}(\varsigma, \iota, \eta(\iota))=\mathbf{E}_{c, d}(\varsigma-\iota) \eta(\iota)$ for every $\varsigma \in \mathcal{G}_{1}$ and $\iota \leq \varsigma$. 
Let $\psi: \mathcal{G}_{1} \times\left(\mathcal{G}_{2}\right)^{4} \rightarrow \operatorname{diag} \mathcal{M}_{4}\left(\mathcal{G}_{3}\right)$ be defined as follows:

$$
\psi(\varsigma, \vec{\zeta})=\operatorname{diag}\left[\mathbf{E}_{\aleph}\left(-\frac{|\zeta|}{\zeta_{1}}\right), \frac{\zeta_{2}}{\zeta_{2}+|\zeta|}, \exp \left(-\frac{|\zeta|}{\zeta_{3}}\right), \mathbf{E}_{\aleph}\left(-\frac{|\zeta|}{\zeta_{4}}\right)\right]
$$

for every $\varsigma \in \mathcal{G}_{1}, \vec{\zeta} \in\left(\mathcal{G}_{2}\right)^{4}$ and $\aleph \in \mathcal{G}_{3}$. Let $\gamma: \mathcal{G}_{1} \rightarrow \mathbb{R}$ be a differentiable function satisfying

$$
\mathscr{S}\left(\gamma(\varsigma)-\ln \sqrt{|\eta(\varsigma)|}-{ }_{0^{+}}^{H} \mathcal{I}_{\phi(\varsigma)}^{\kappa, \vartheta} \mathbf{E}_{c, d}(\varsigma-\iota) \gamma(\iota), \vec{\zeta}\right) \succeq \psi(\varsigma, \vec{\zeta})
$$

for every $\varsigma \in \mathcal{G}_{1}$ and $\vec{\zeta} \in\left(\mathcal{G}_{2}\right)^{4}$. Now Theorem 4.1 implies that, if $\max \left[\complement, \Lambda \Theta_{4}\right]<0.5$, we can find a unique differentiable function $\gamma_{0}: \mathcal{G}_{1} \rightarrow \mathbb{R}$ such that

$$
\gamma_{0}(\varsigma)=\ln \sqrt{|\eta(\varsigma)|}+{ }_{0^{+}}^{H} \mathcal{I}_{\phi\left(\mathcal{G}_{1}\right)}^{\kappa, \vartheta} \mathbf{E}_{c, d}(\varsigma-\iota) \gamma(\iota)
$$

and

$$
\mathscr{S}\left(\gamma(\varsigma)-\gamma_{0}(\varsigma), \vec{\zeta}\right) \succeq \psi\left(\varsigma, \frac{\left(1-2 \max \left[\complement, \Lambda \Theta_{4}\right]\right) \vec{\zeta}}{\max \left[1, \Theta_{4}\right]}\right)
$$

for every $\varsigma \in \mathcal{G}_{1}$ and $\vec{\zeta} \in\left(\mathcal{G}_{2}\right)^{4}$.

\section{Conclusions}

In this paper, we presented an example of fuzzy normed space by means of the MittagLeffler function. Next, we extended the concept of fuzzy normed space to a matrix valued fuzzy normed space and also we applied the Alternative fixed point theorem to investigating Ulam-Hyers-Rassias stability of some fractional equations in MVFB-spaces. We defined a class of matrix valued fuzzy control functions for stabilizing both the $\phi$-Hadamard fractional Volterra integro-differential equation and the $\phi$-Hadamard fractional Volterra integral equation in MVFB-spaces and we have obtained best approximation for this kind of fractional equations. Finally, as an application, we investigated the Ulam-HyersRassias stability using a matrix valued fuzzy control function obtained through the MittagLeffler function.

Acknowledgements

The authors are thankful to the area editor and referees for giving valuable comments and suggestions.

Funding

No funding.

Availability of data and materials

Not applicable.

Competing interests

The authors declare that they have no competing interests.

Authors' contributions

All authors conceived of the study, participated in its design and coordination, drafted the manuscript, participated in the sequence alignment, and read and approved the final manuscript. 


\section{Publisher's Note}

Springer Nature remains neutral with regard to jurisdictional claims in published maps and institutional affiliations.

Received: 9 January 2021 Accepted: 15 February 2021 Published online: 04 March 2021

\section{References}

1. Almeida, R., Tavares, D., Torres, D.F.M.: The Variable-Order Fractional Calculus of Variations. Springer Briefs in Applied Sciences and Technology. Springer, Cham (2019)

2. El-Sayed, A.M.A., Gaafar, F.M.: Existence and uniqueness of solution for Sturm-Liouville fractional differential equation with multi-point boundary condition via Caputo derivative. Adv. Differ. Equ. 2019, 46 (2019)

3. Jiang, J., O'Regan, D., Xu, J., Fu, Z.: Positive solutions for a system of nonlinear Hadamard fractional differential equations involving coupled integral boundary conditions. J. Inequal. Appl. 2019, 204 (2019)

4. Hadzic, O., Pap, E.: Fixed Point Theory in Probabilistic Metric Spaces. Mathematics and Its Applications, vol. 536. Kluwer Academic, Dordrecht (2001)

5. Klement, E.P., Mesiar, R., Pap, E.: Triangular Norms. Trends in Logic-Studia Logica Library, vol. 8. Kluwer Academic, Dordrecht (2000)

6. Schweizer, B., Sklar, A.: Probabilistic Metric Spaces. North-Holland Series in Probability and Applied Mathematics. North-Holland, New York (1983)

7. Šerstnev, A.N.: On the notion of a random normed space. Dokl. Akad. Nauk SSSR 149, 280-283 (1963)

8. Pap, E., Park, C., Saadati, R.: Additive $\sigma$-random operator inequality and rhom-derivations in fuzzy Banach algebras. Sci. Bull. "Politeh." Univ. Buchar., Ser. A, Appl. Math. Phys. 82, 3-14 (2020)

9. Constantinescu, C.D., Ramirez, J.M., Zhu, W.R.: An application of fractional differential equations to risk theory. Finance Stoch. 23, 1001-1024 (2019)

10. Asaduzzaman, M., Kilicman, A., Ali, M.Z.: Presence and diversity of positive solutions for a Caputo-type fractional order nonlinear differential equation with an advanced argument. J. Math. Comput. Sci. 23, 230-244 (2021)

11. Chaharpashlou, R., Saadati, R., Atangana, A.: Ulam-Hyers-Rassias stability for nonlinear $\Psi$-Hilfer stochastic fractional differential equation with uncertainty. Adv. Differ. Equ. 2020, 339 (2020)

12. Madadi, M., Saadati, R., Park, C., Rassias, J.M.: Stochastic Lie bracket (derivation, derivation) in MB-algebras. J. Inequal. Appl. 2020, 141 (2020)

13. Jung, S., Rassias, M.T., Mortici, C.: On a functional equation of trigonometric type. Appl. Math. Comput. 252, 294-303 (2015)

14. Park, C., Rassias, M.T.: Additive functional equations and partial multipliers in $C^{*}$-algebras. Rev. R. Acad. Cienc. Exactas Fís. Nat., Ser. A Mat. 113, 2261-2275 (2019)

15. Cădariu, L., Radu, V.: Fixed points and the stability of Jensen's functional equation. J. Inequal. Pure Appl. Math. 4, 1 (2003)

16. Diaz, J.B., Margolis, B.: A fixed point theorem of the alternative, for contractions on a generalized complete metric space. Bull. Am. Math. Soc. 74, 305-309 (1968)

17. Sousa, J.V. da C., Fabio, G.R., de Oliveira, E.C.: Stability of the fractional Volterra integro-differential equation by means of $\Psi$-Hilfer operator. Math. Methods Appl. Sci. 42, 3033-3043 (2019)

18. El-Sayed, A.M.A., Al-Issa, S.M.: Existence of integrable solutions for integro-differential inclusions of fractional order; coupled system approach. J. Nonlinear Sci. Appl. 13, 180-186 (2020)

19. Khan, O., Araci, S., Saif, M.: Fractional calculus formulas for Mathieu-type series and generalized Mittag-Leffler function. J. Math. Comput. Sci. 20, 122-130 (2020)

20. Sene, N.: Global asymptotic stability of the fractional differential equations. J. Nonlinear Sci. Appl. 13, 171-175 (2020)

21. Cădariu, L., Radu, V.: Fixed point methods for the generalized stability of functional equations in a single variable. Fixed Point Theory Appl. 2008, Article ID 749392 (2008)

22. Miheț, D., Radu, V:: On the stability of the additive Cauchy functional equation in random normed spaces. J. Math. Anal. Appl. 343, 567-572 (2008)

\section{Submit your manuscript to a SpringerOpen ${ }^{\circ}$ journal and benefit from:}

- Convenient online submission

- Rigorous peer review

- Open access: articles freely available online

- High visibility within the field

- Retaining the copyright to your article

Submit your next manuscript at $\downarrow$ springeropen.com 